\title{
HYDROCARBON EMISSION RINGS IN PROTOPLANETARY DISKS INDUCED BY DUST EVOLUTION
}

\author{
Edwin A. Bergin ${ }^{1}$, Fujun Du ${ }^{1}$, L. Ilsedore Cleeves $^{2}$, G. A. Blake ${ }^{3}$, K. Schwarz ${ }^{1}$, R. Visser ${ }^{4}$, And K. Zhang ${ }^{1}$ \\ ${ }^{1}$ Department of Astronomy, University of Michigan, 311 West Hall, 1085 S. University Ave, Ann Arbor, MI 48109, USA \\ ${ }^{2}$ Harvard-Smithsonian Center for Astrophysics, 60 Garden Street, Cambridge, MA 02138, USA \\ ${ }^{3}$ Division of Geological \& Planetary Sciences, MC 150-21, California Institute of Technology, 1200 E California Blvd, Pasadena, CA 91125, USA \\ ${ }^{4}$ European Southern Observatory, Karl-Schwarzschild-Str. 2, D-85748, Garching, Germany \\ Received 2016 May 6; revised 2016 September 9; accepted 2016 September 20; published 2016 October 31
}

\begin{abstract}
We report observations of resolved $\mathrm{C}_{2} \mathrm{H}$ emission rings within the gas-rich protoplanetary disks of TW Hya and DM Tau using the Atacama Large Millimeter Array. In each case the emission ring is found to arise at the edge of the observable disk of millimeter-sized grains (pebbles) traced by submillimeter-wave continuum emission. In addition, we detect a $\mathrm{C}_{3} \mathrm{H}_{2}$ emission ring with an identical spatial distribution to $\mathrm{C}_{2} \mathrm{H}$ in the TW Hya disk. This suggests that these are hydrocarbon rings (i.e., not limited to $\mathrm{C}_{2} \mathrm{H}$ ). Using a detailed thermo-chemical model we show that reproducing the emission from $\mathrm{C}_{2} \mathrm{H}$ requires a strong UV field and $\mathrm{C} / \mathrm{O}>1$ in the upper disk atmosphere and outer disk, beyond the edge of the pebble disk. This naturally arises in a disk where the ice-coated dust mass is spatially stratified due to the combined effects of coagulation, gravitational settling and drift. This stratification causes the disk surface and outer disk to have a greater permeability to UV photons. Furthermore the concentration of ices that transport key volatile carriers of oxygen and carbon in the midplane, along with photochemical erosion of $\mathrm{CO}$, leads to an elemental $\mathrm{C} / \mathrm{O}$ ratio that exceeds unity in the UV-dominated disk. Thus the motions of the grains, and not the gas, lead to a rich hydrocarbon chemistry in disk surface layers and in the outer disk midplane.
\end{abstract}

Key words: astrochemistry - circumstellar matter - molecular processes - planet-disk interactions - planetary systems - planets and satellites: atmospheres

\section{INTRODUCTION}

The birth of planetary systems begins with the gravitational collapse of a centrally concentrated core in moleculedominated gas that forms a star and disk system. Over time the energy released by the forming star, both dynamical and radiative, ablates the surrounding envelope, exposing the dense $\left(n_{\mathrm{H}_{2}} \gg 10^{5} \mathrm{~cm}^{-3}\right)$ disk to interstellar space. The gas and dust rich protoplanetary disk then continues to evolve both physically and chemically until the gaseous disk dissipates and the system transitions to one dominated by large bodies and their surrounding debris (so-called debris disk systems).

A key facet of the disk evolution is the growth of the initially micron-sized dust particles to larger sizes, in which two aspects stand out. First, the gravitational settling of coagulating grains to the midplane removes dust from the surface layers of the disk (Weidenschilling \& Cuzzi 1993; Dullemond \& Dominik 2004). This process is constrained via observations of the dust spectral energy distribution because dust settling decreases continuum emission in the mid- to far-infrared (D'Alessio et al. 1999; Chiang et al. 2001). Surveys using the Spitzer Space Telescope infer dust depletion factors in the surface layers to be of the order of 100-1000 (relative to what is expected assuming interstellar grain abundances) in both Taurus and Ophiuchus (Furlan et al. 2006; McClure et al. 2010). Second, dust becomes radially stratified by size-dependent gas drag, a process known as radial drift (Whipple 1972; Weidenschilling \& Cuzzi 1993; Youdin \& Kenyon 2013). Small grains are coupled to gas motions while large kilometer-sized planetesimals have significant inertia to resist the overall drag force. In between, millimeter to tens of $\mathrm{cm}$-sized grains (Youdin \& Kenyon 2013) radially drift inwards unless subject to a local pressure maximum generated by a variety of mechanisms (Johansen et al. 2007; Chiang \& Youdin 2010). There is now growing observational evidence for the pervasive presence of radial drift; one example is that the part of a disk composed of millimeter-sized grains seen in submillimeter emission is smaller in size than the part composed of smaller grains traced by scattered light or ${ }^{12} \mathrm{CO}$ emission (Isella et al. 2007; Piétu et al. 2007; Panić et al. 2009; Andrews et al. 2012).

What is less clear is how the evolution of dust affects the gas, and in particular the gas chemical composition. The settling and growth of dust grains increases the transparency of the surface layers to UV radiation, while also increasing the local timescales for the freeze-out of the gas species (Aikawa et al. 1999; Aikawa \& Nomura 2006; Bethell \& Bergin 2011; Fogel et al. 2011; Semenov \& Wiebe 2011; Akimkin et al. 2013). However, the effects are difficult to disentangle, and, while many chemical dependencies have been found in the models, strong correlations are yet to be discovered.

More recently, there is observational evidence that the abundances of key volatile species are depleted in the surface layers where the molecular emission originates-the so-called "warm molecular layer" (Aikawa et al. 2002). The generic expectation is that $\mathrm{CO}$ would be present as vapor with ISM abundance $\left(\sim 10^{-4}\right.$ relative to $\left.\mathrm{H}_{2}\right)$ in layers where the dust temperature exceeds $\sim 20 \mathrm{~K}$. In gas below $20 \mathrm{~K}$, low gas-phase $\mathrm{CO}$ abundances relative to the ISM are a hallmark of volatile freeze-out, and are seen clearly in observations of the cold regions of protoplanetary disks (Dutrey et al. 1997). However, Dutrey et al. (2003) and Chapillon et al. (2008) find that the gas-phase abundance of $\mathrm{CO}$ may also be reduced in layers above the sublimation temperature. A similar result is found for water vapor emitting from cold $(\sim 20-40 \mathrm{~K})$ layers well below the sublimation temperature (Bergin et al. 2010; Hogerheijde et al. 2011). Still, molecular depletion is not the only explanation for the observed low volatile abundance in warm 
Table 1

Hydrocarbon Observations

\begin{tabular}{|c|c|c|c|}
\hline Transitions & $\begin{array}{l}\text { Frequency } \\
\qquad(\mathrm{GHz})\end{array}$ & $\begin{array}{l}\text { Peak Flux } \\
\left(\text { Jy beam }^{-1}\right)\end{array}$ & $\begin{array}{l}\text { Disk-integrated Flux } \\
\left(\mathrm{Jy} \mathrm{km} \mathrm{s}^{-1}\right)\end{array}$ \\
\hline \multicolumn{4}{|l|}{ TW Hya $(0 " .48 \times 0 . " 39) \mathrm{PA}=57.8$} \\
\hline \multicolumn{4}{|l|}{$\mathrm{C}_{2} \mathrm{H}$} \\
\hline$N=4-3, J=9 / 2-7 / 2, F=4-4$ & $349.313 \mathrm{GHz}$ & $0.660 \pm 0.007$ & $0.20 \pm 0.02$ \\
\hline$N=4-3, J=9 / 2-7 / 2, F=5-4$ & $349.338 \mathrm{GHz}$ & $1.100 \pm 0.007$ & $12.14 \pm 0.04^{\dagger}$ \\
\hline$N=4-3, J=9 / 2-7 / 2, F=4-3$ & $349.339 \mathrm{GHz}$ & $1.002 \pm 0.007$ & \\
\hline$N=4-3, J=7 / 2-5 / 2, F=4-3$ & $349.399 \mathrm{GHz}$ & $0.862 \pm 0.007$ & $10.72 \pm 0.04^{\mathrm{b}}$ \\
\hline$N=4-3, J=7 / 2-5 / 2, F=3-2$ & $349.401 \mathrm{GHz}$ & $0.866 \pm 0.007$ & \\
\hline$N=4-3, J=7 / 2-5 / 2, F=3-3$ & $349.415 \mathrm{GHz}$ & $0.089 \pm 0.007$ & $0.47 \pm 0.02$ \\
\hline \multicolumn{4}{|l|}{$c-\mathrm{C}_{3} \mathrm{H}_{2}^{\mathrm{a}}$} \\
\hline$\left(J_{K^{+}, K^{-}}\right) 10_{1,10}-9_{0,9} / 10_{0,10}-9_{1,9}$ & $351.782 \mathrm{GHz}$ & $0.108 \pm 0.007$ & $0.37 \pm 0.02$ \\
\hline$\left(J_{K^{+}, K^{-}}\right) 9_{1,8}-8_{2,7} / 9_{2,8}-8_{1,7}$ & $351.966 \mathrm{GHz}$ & $0.079 \pm 0.007$ & $0.32 \pm 0.02$ \\
\hline$\left(J_{K^{+}, K^{-}}\right) 8_{3,6}-7_{2,5} / 8_{2,6}-7_{3,5}$ & $352.194 \mathrm{GHz}$ & $0.067 \pm 0.007$ & $0.23 \pm 0.02$ \\
\hline$\left(J_{K^{+}, K^{-}}\right) 5_{5,1}-4_{4,0}$ & $338.204 \mathrm{GHz}$ & $0.066 \pm 0.007$ & $0.14 \pm 0.02$ \\
\hline \multicolumn{4}{|l|}{ DM Таи $(0 "$ " $56 \times 0$ " 37$) \mathrm{PA}=-28.3$} \\
\hline$N=3-2, J=7 / 2-5 / 2, F=3-3$ & $261.978 \mathrm{GHz}$ & $\lesssim 0.003$ & $\ldots$ \\
\hline$N=3-2, J=7 / 2-5 / 2, F=4-3$ & $262.004 \mathrm{GHz}$ & $0.066 \pm 0.003$ & $2.00 \pm 0.07^{\mathrm{b}}$ \\
\hline$N=3-2, J=7 / 2-5 / 2, F=3-2$ & $262.006 \mathrm{GHz}$ & $0.063 \pm 0.003$ & \\
\hline$N=3-2, J=5 / 2-3 / 2, F=3-2$ & $262.065 \mathrm{GHz}$ & $0.058 \pm 0.003$ & $1.49 \pm 0.07^{\mathrm{b}}$ \\
\hline$N=3-2, J=5 / 2-3 / 2, F=2-1$ & $262.067 \mathrm{GHz}$ & $0.048 \pm 0.003$ & \\
\hline$N=3-2, J=5 / 2-3 / 2, F=2-2$ & $262.079 \mathrm{GHz}$ & $\lesssim 0.003$ & $\cdots$ \\
\hline
\end{tabular}

Notes.

a In some cases detected lines are blends of degenerate transitions.

b The central hyperfine components are partially blended, so the reported disk-integrated fluxes are the sum of the indicated line pairs.

gas; the disk could also simply be less massive than the existing mass tracers predict.

The degeneracy between abundance and gas mass can be minimized by using a gas tracer, such as $\mathrm{HD}$, to infer the $\mathrm{H}_{2}$ mass (Bergin et al. 2013). Favre et al. (2013) use $\mathrm{C}^{18} \mathrm{O}$ and HD emission to show that the $\mathrm{CO}$ abundance was significantly reduced in emissive layers in the TW Hya disk, while Du et al. (2015) find that both CO and water appear to be depleted from the surface beyond their respective snow lines. They hypothesize, in concert with earlier and contemporary work (Chapillon et al. 2008; Hogerheijde et al. 2011; Kama et al. 2016a, 2016b), that the ice-aided growth (Gundlach \& Blum 2015) and motions of grains deplete the upper layers of volatile species. Taking into account this effect, Du et al. (2015) are able to match the observed emission from $\mathrm{CII}, \mathrm{CI}, \mathrm{CO}, \mathrm{O}$, $\mathrm{OH}$, and $\mathrm{H}_{2} \mathrm{O}$; they also predict that regions of the disk surface and outer radii will be preferentially depleted in oxygen and will become subsequently hydrocarbon-rich. This composition arises because $\mathrm{C}$ - and $\mathrm{N}$-bearing molecules exist in a sea of $\mathrm{H}_{2}$ rich gas that is exposed to ionizing photons initiating hydrocarbon formation. Contemporaneously Kastner et al. (2015), using the SMA, detected the presence of a bright $\mathrm{C}_{2} \mathrm{H}$ emission ring in TW Hya.

Here we present the spatially resolved distribution of $\mathrm{C}_{2} \mathrm{H}$ and $\mathrm{C}_{3} \mathrm{H}_{2}$ emission in TW Hya and $\mathrm{C}_{2} \mathrm{H}$ in DM Tau obtained with the Atacama Large Millimeter/submillimeter Array (ALMA). Using the thermo-chemical model of Du \& Bergin (2014), we demonstrate that hydrocarbon rings are a natural feature induced by the settling and drift of millimeter or larger sized grains, which removes UV opacity from the upper layers and outer disk, creating a UV-dominated disk (essentially a photon dominated region, which is often abbreviated as PDR). The chemical outcome of this drift is to deplete the upper layers of oxygen and raise the $\mathrm{C} / \mathrm{O}$ ratio there. This is also required to boost hydrocarbon emission in the ring to the detected levels. In Section 2 we describe the observational setup and in Section 3 we present images of the two sources. In Section 4 we use the thermo-chemical model of Du \& Bergin (2014) and $\mathrm{Du}$ et al. (2015) to outline the ingredients required to produce hydrocarbon rings. In Section 5, we present source-specific models that reproduce the observed emission, compare these predictions with an excitation analysis of TW Hya, and discuss potentials sources for the active carbon chemistry. Finally, Section 6 summarizes these results and discusses the implications.

\section{OBSERVATIONS}

Observations of the DM Tau and TW Hya protoplanetary disk systems were acquired with the ALMA, under the project code ADS/JAO.ALMA\#2013.1.00198.S. DM Tau was observed on 2015 June 6 with 37 antennas (21.4-740.4 m baselines) and on 2014 December 25 with 38 antennas (15.1-348.5 m baselines). TW Hya was observed on 2014 December 31 and 2015 June 15 with 34 antennas (15.1-348.5 $\mathrm{m}$ baselines) and 36 antennas (21.36-783.55 m baselines), respectively. The total on-source integration time for DM Tau in both configurations was 57.1 minutes. The total on-source integration time for TW Hya was 43.4 minutes. For DM Tau, the nearby quasar J0510+180 was used for gain and absolute flux calibration. Quasar J0423-0120 was used for bandpass calibration. For TW Hya, the quasars J1256-057 and J1037-2934 were used for bandpass and gain calibration, respectively. Titan was used for the flux calibration.

The spectral setup targeted the $\mathrm{C}_{2} \mathrm{H} N=3-2$ hyperfine complex in DM Tau and $\mathrm{C}_{2} \mathrm{H} N=4-3$ and multiple 
a) TW Hya $\mathrm{C}_{2} \mathrm{H} \quad \mathrm{N}=4-3$

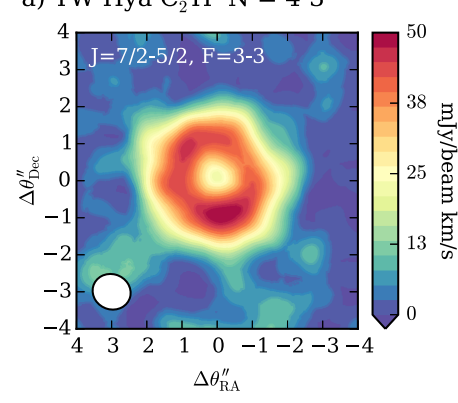

b) DM Tau $\mathrm{C}_{2} \mathrm{H} \quad \mathrm{N}=3-2$
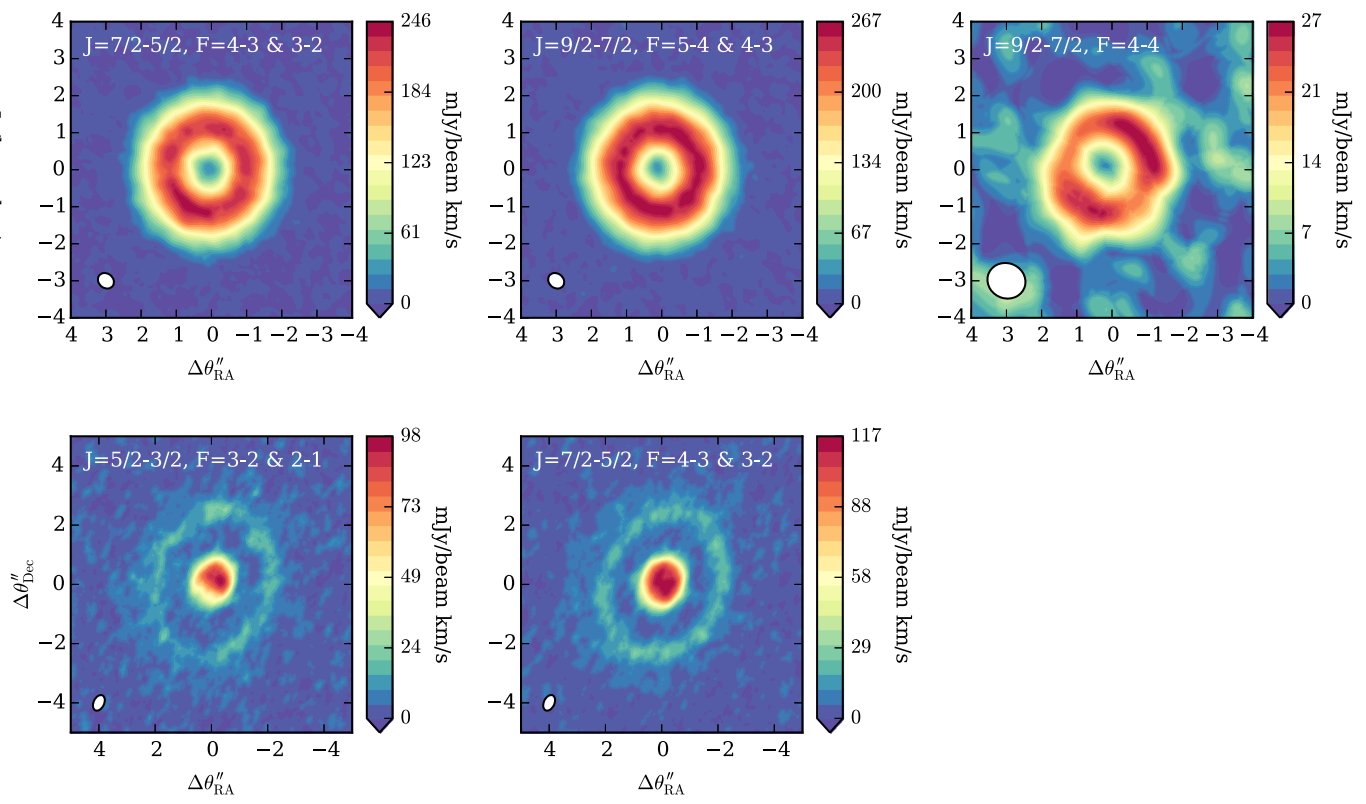

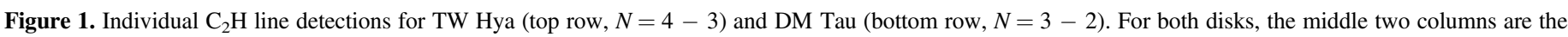

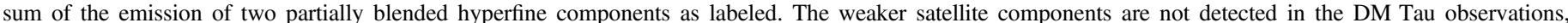

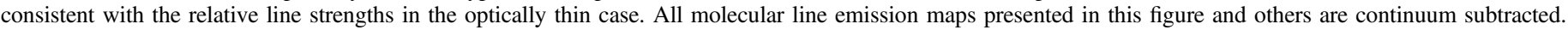

transitions of $c-\mathrm{C}_{3} \mathrm{H}_{2}$ in TW Hya (see Table 1). Observations of both sources were carried out with $122.07 \mathrm{kHz}$ width channels. From the pipeline calibrated measurement sets we selfcalibrated the data using each source's bright continuum emission to improve the signal-to-noise. There was a dedicated continuum spectral window in the same sideband as the DM Tau $\mathrm{C}_{2} \mathrm{H}$ emission, which we use for the self-calibration. For the TW Hya data set, there was a pointing misalignment that may in part be due to TW Hya's high proper motion, and so we aligned the compact and extended data's phase-centers based on the continuum peak location. TW Hya's dedicated continuum window was in the opposite sideband from the $\mathrm{C}_{2} \mathrm{H}$ lines, so we instead used the continuum data from the spectral window containing the lines, which were masked. We imaged the measurement sets with the clean task in CASA version 4.3 using Briggs' weighting with a robust parameter of 0.5 . For DM Tau, the resulting rms noise level in the image is $2.5 \mathrm{mJy} \mathrm{beam}^{-1}$ in $0.279 \mathrm{~km} \mathrm{~s}^{-1}$, and for TW Hya is $7 \mathrm{mJy}_{\text {beam }}^{-1}$ in $0.15 \mathrm{~km} \mathrm{~s}^{-1}$ channels. The peak and disk integrated fluxes are reported in Table 1 with rms uncertainties. An additional 15\% uncertainty should be applied for the absolute flux calibration uncertainty. The synthesized beam for the DM Tau combined data sets has a shape and position angle (PA) of $0 . " 56 \times 0$. . 37 and PA $=-28^{\circ} .3$, and for TW Hya, 0 " $48 \times 0$ ". 39 and PA $=57^{\circ} .8$. The moment 0 maps for the individual line detections for both sources are shown in Figure 1.

We have stacked the individual lines to create a high signalto-noise spectrally integrated map of the $\mathrm{C}_{2} \mathrm{H}$ emission morphology, shown in Figure 2. For both disks, we derive a disk position angle and inclination from the outer $\mathrm{C}_{2} \mathrm{H}$ ring, which we use to deproject the image to create a disk brightness profile. The outer ring in DM Tau has a position angle of $157 \pm 5^{\circ}$ and inclination of $i=36 \pm 3^{\circ}$. The ring in TW Hya has a position angle of $150 \pm 10^{\circ}$ and inclination of $i=7 \pm 4^{\circ}$. The spectrally integrated line and continuum emission profiles are shown in Figure 2, bottom row.

In the stacked profile, the inner $\mathrm{C}_{2} \mathrm{H}$ emission in the DM Tau disk flattens out toward the center. This flattening motivated us to reimage DM Tau using extended data only, with uniform weighting to optimize the image resolution. The beam on the higher resolution images is 0 ." $30 \times 0$." $48, \mathrm{PA}=-23^{\circ} .8$. With this smaller beam, we successfully resolve a second ring in DM Tau, shown in Figure 3. The rms noise on the high resolution image is $6 \mathrm{mJy} \mathrm{beam}^{-1}$ in $0.279 \mathrm{~km} \mathrm{~s}^{-1}$ channels, and $8 \mathrm{mJy} /$ beam $\mathrm{km} \mathrm{s}^{-1}$ on the spectrally integrated image. We are unable to resolve the previously reported $R=20$ au inner hole in the continuum (Andrews et al. 2011), even with the higher resolution image. However, detailed modeling of the continuum visibilities of the same data by Zhang et al. (2016) shows evidence for a break in the inner disk on a similar scale.

\section{HYDROCARBON RINGS}

\section{1. $\mathrm{C}_{2} \mathrm{H}$ Emission Distributions in TW Hya and DM Tau}

Both TW Hya and DM Tau are well studied sources. TW Hya is the closest young star with a disk at a distance of $54 \pm 6$ pc (van Leeuwen 2007). Its ${ }^{12} \mathrm{CO}$ molecular distribution covers the full extent of the disk seen in scattered light (Weinberger et al. 2002; Andrews et al. 2012). Based on a search for $\mathrm{CO}$ emission and $\mathrm{Na}$ I absorption, the surrounding stellar association appears to be unassociated with its parent molecular cloud (Tachihara et al. 2009). Furthermore, the extinction to this nearly face-on disk system is nearly zero, as demonstrated by the detection of almost its entire UV spectrum down to near the Lyman limit (Herczeg et al. 2002). There is some controversy in the current literature regarding its spectral type and age, which is discussed in detail by Debes et al. (2013) and Vacca \& Sandell (2011), with the spectral range from $\mathrm{K} 7$ to $\mathrm{M} 2$ and an age of $8 \pm 4 \mathrm{Myr}$. 

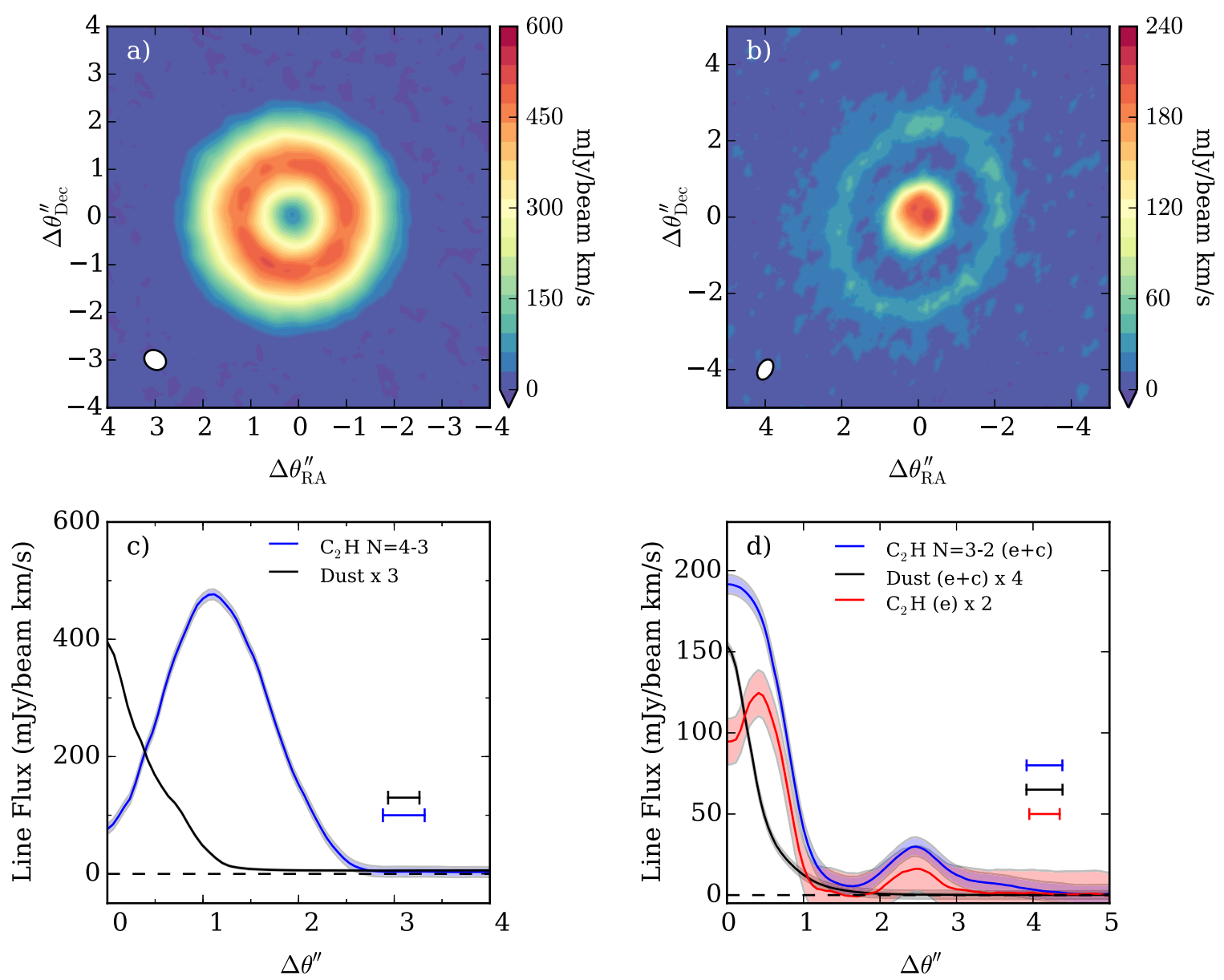

Figure 2. Top row: stacked image of the individual $\mathrm{C}_{2} \mathrm{H}$ detections presented in Figure 1 for (a) TW Hya and (b) DM Tau. Bottom row: deprojected line and continuum emission profile for (c) TW Hya and (d) DM Tau. The TW Hya image profile is made from the compact TW Hya data for the $\mathrm{C}_{2} \mathrm{H} N=4-3$ (blue) and continuum (black). The DM Tau emission profile is made from combined compact and extended data for the $\mathrm{C}_{2} \mathrm{H} N=3-2$ line (blue) and continuum (black). The DM Tau $\mathrm{C}_{2} \mathrm{H}$ data re-imaged with higher resolution weighting and extended data only is shown in red. Continuum profiles are in units of mJy beam ${ }^{-1}$.

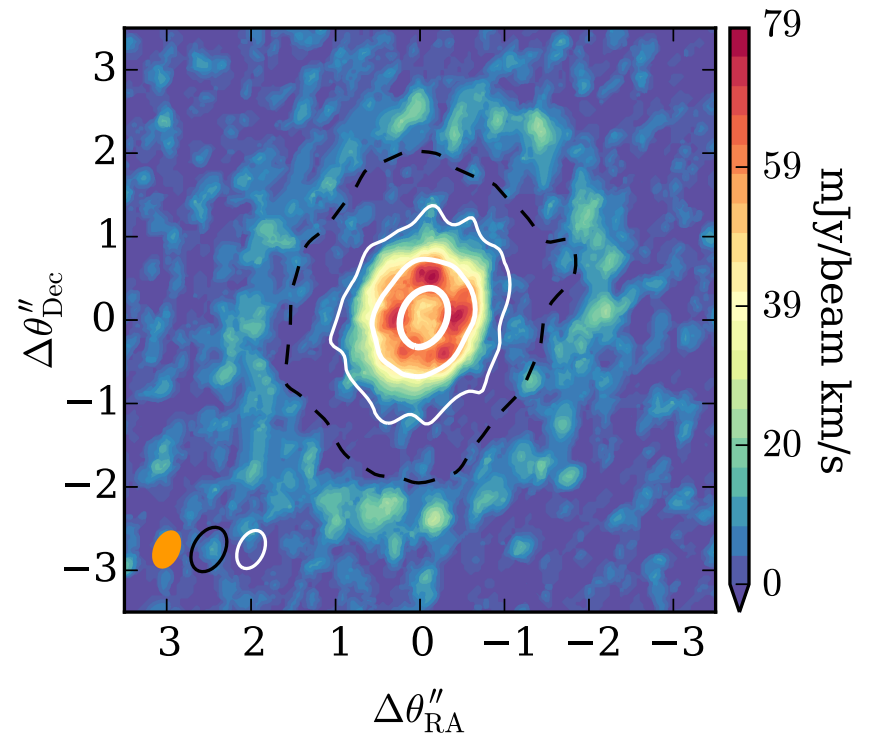

Figure 3. High resolution DM Tau image using the extended data only, where the inner emission peak is resolved into a second ring. Overlaid are continuum contours, where the black dashed line shows the $3 \sigma$ continuum contour for the compact and extended data. The white contours highlight the 6,24 , and $96 \sigma$ continuum contours for the extended data only, going from thin to thick line weights. The inner dust emission is extremely steeply peaked at the location of the $\mathrm{C}_{2} \mathrm{H}$ inner ring.
DM Tau is an M1 star surrounded by a $\gtrsim 1000$ au molecular disk as seen in the emission of ${ }^{12} \mathrm{CO}$ (Simon et al. 2000; Dartois et al. 2003). It is located near the edge of the of the Taurus Molecular Cloud (Hartmann et al. 2001; Goldsmith et al. 2008) at a distance of $\sim 140 \mathrm{pc}$ (Kenyon et al. 1994; Schlafly et al. 2014), where it may be partially obscured by cloud material ( $A_{V} \sim 0$. 7 ; Espaillat et al. 2010). Based on its spectral energy distribution and high resolution submillimeter dust continuum imaging, it is also a transition disk with a measured (submillimeter emission) inner gap radius of 19 au (Calvet et al. 2005; Andrews et al. 2011). The age of the system is estimated using models of pre-main sequence evolution with some uncertainty. Hartmann et al. (1998) placed DM Tau on the center of the age distribution in Taurus (1-2 Myr) at $1.5 \mathrm{Myr}$; but other work suggests it is slightly older ( 2-4.5 Myr; Kitamura et al. 2002; Hueso \& Guillot 2005). At face value DM Tau appears younger than TW Hya. This is based on the models of Hayashi track evolution discussed above, but also from the fact that molecular cloud material is present in close proximity of DM Tau, while absent near the TW Hya association.

Based on Figure 2, the dust emission at submillimeter wavelengths is confined with respect to the $\mathrm{C}_{2} \mathrm{H}$ emission for TW Hya. The continuum emission at $\sim 850 \mu \mathrm{m}$ originates from dust grains with sizes up to $\sim$ millimeter (Draine 2006). In TW Hya the $\mathrm{C}_{2} \mathrm{H}$ emission peaks near the edge of the disk traced by 


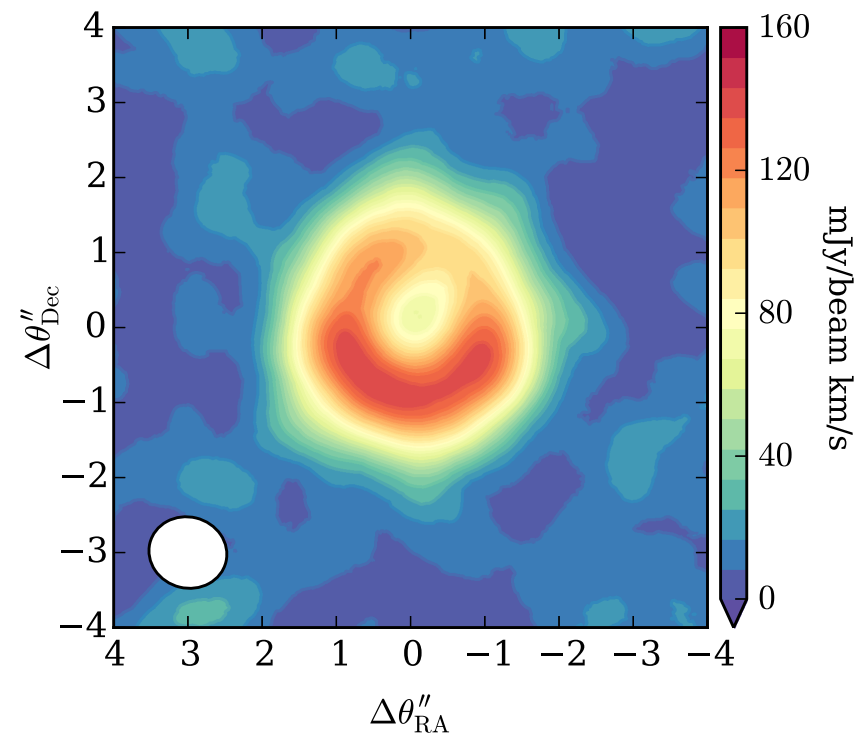

Figure 4. Stacked image of all detected transitions of $c-\mathrm{C}_{3} \mathrm{H}_{2}$ toward TW Hya. The transitions are listed in Table 1.

submillimeter emission, down to our sensitivity limit. A recent paper by Hogerheijde et al. (2016) traces the edge of the surface density distribution at $820 \mu \mathrm{m}$ to a radius of $47.1 \pm 0.2 \mathrm{au}\left(0\right.$ !" 87). The peak of the $\mathrm{C}_{2} \mathrm{H}$ ring appears just beyond this edge at $R \sim 1$ !' 1 , with a well resolved, deep central depression.

In DM Tau there is significantly more structure in the $\mathrm{C}_{2} \mathrm{H}$ emission. In Figure 3 we show the $\mathrm{C}_{2} \mathrm{H}$ emission distribution using only the extended baselines. Overlaid on this image is the dust continuum emission with the black line showing the $3 \sigma$ dust emission edge at $1100 \mu \mathrm{m}$. At the peak of the dust emission there is a central hole in the $\mathrm{C}_{2} \mathrm{H}$ emission that encompasses the inner $100 \mathrm{au}$ (diameter). This is followed by a ring of $\mathrm{C}_{2} \mathrm{H}$ emission, also with a width $\sim 100 \mathrm{au}$ where the strongest emission is detected. At much greater distances ( $R \sim 350 \mathrm{au}$ ) there is a weaker and thinner $\mathrm{C}_{2} \mathrm{H}$ emission ring. The fact that both TW Hya and DM Tau have hydrocarbon ring emission near the millimeter-dust edge is highly suggestive of a common process that might be present in evolving disk systems. However, the large differences in the size scale of the rings, along with stronger central emission in DM Tau, requires detailed modeling to determine the import of specific mechanisms.

\section{2. c- $\mathrm{C}_{3} \mathrm{H}_{2}$ Ring in $\mathrm{TW} \mathrm{Hya}$}

Similar to Kastner et al. (2015), we mainly focus on the detection of $\mathrm{C}_{2} \mathrm{H}$ rings in this paper. In Figure 4 we present stacked observations of $c-\mathrm{C}_{3} \mathrm{H}_{2}$ toward TW Hya that clearly show a ring with similar dimensions as $\mathrm{C}_{2} \mathrm{H}$. Thus, the detection of rings is not limited to $\mathrm{C}_{2} \mathrm{H}$ alone. Instead we will argue below that it likely extends toward all hydrocarbons, as supported by the $c-\mathrm{C}_{3} \mathrm{H}_{2}$ detection in TW Hya. ${ }^{5}$

\subsection{Dust Optical Depth and Ring Structure}

High dust optical depth is one mechanism that would produce inner holes in the emission of molecular tracers at

\footnotetext{
5 The $\mathrm{C}_{3} \mathrm{H}_{2}$ data will be analyzed in a separate work (I. Cleeves et al. 2016, in preparation).
}

these wavelengths. For TW Hya, detailed modeling of the submillimeter continuum emission suggests the inner $\sim 40 \mathrm{au}$ has $\tau>0.5$ (Hogerheijde et al. 2016). Thus only the inner tens of au could be optically thick. In the high resolution study of Andrews et al. (2016), they discuss a possible transition to an optically thick disk near $20 \mathrm{au}$; the modeling of Tsukagoshi et al. (2016) also suggests that beyond $20 \mathrm{au}$, the disk is optically thin at these wavelengths. This is important as the $\mathrm{C}_{2} \mathrm{H}$ emission begins to significantly decline inwards of $60 \mathrm{au}$ -where the dust is in these analysis is optically thin. Kama et al. (2016b) present another recent model of TW Hya and this model is also optically thin where $\mathrm{C}_{2} \mathrm{H}$ emission begins to decline. Thus high dust optical depth is not enough to explain the inner hole and a chemical effect is needed (see also Kastner et al. 2015).

DM Tau has evidence for two $\mathrm{C}_{2} \mathrm{H}$ emission rings, an inner and an outer. Figure 3 shows the contours of the strongly centrally peaked dust emission; nearly all the strongest dust emission $(>6 \sigma)$ is confined within the main central bulge of the $\mathrm{C}_{2} \mathrm{H}$ emission. Here the drop of the $\mathrm{C}_{2} \mathrm{H}$ emission toward the central hole is about a factor of two. At face value this is consistent with what would be expected from the effects of high dust optical depth, as we would not detect the back side of the disk. Thus, at this resolution we cannot determine whether the central depression is a true chemical effect. However, the outer ring appears at the edge of the dust continuum emission which must be optically thin at that radial distance. The contrast between the $\mathrm{C}_{2} \mathrm{H}$ peak at $(\sim 2$ !" 4$)$ and trough $(\sim 1$ !" 5$)$ is also $\geqslant 3$. Thus, the outer ring is not an effect of dust optical depth and is the result of a change in molecular abundance.

\section{PHYSICAL AND CHEMICAL MODELS}

In the following we will explore the physical/chemical structure within a realistic disk model framework to detail the origin of the ring structure and how to reproduce the hydrocarbon emission. For this purpose we will use the disk model described by $\mathrm{Du}$ et al. (2015) with basic parameters for TW Hya and DM Tau given in Table 2. For the dust composition, we use an 8:2 mixture of astrosilicates and graphite. Their optical constants are taken from Draine \& Lee (1984). ${ }^{6}$ The dust size distribution is a power-law with exponent 3.5 (Mathis et al. 1977), and each disk component has different minimum and maximum grain sizes. A uniform gas-to-dust mass ratio of 100 is used over the whole disk such that $\rho_{\text {gas }}(r, z)=100 \times \rho_{\text {dust }}^{\text {tot }}(r, z)$, regardless of the dust components. We assume three components for both disks: an optically thin inner zone, a submillimeter intermediate one (Andrews et al. 2012; Menu et al. 2014; Hogerheijde et al. 2016), and a UV-dominated outer region. The disk parameterization is the same as Andrews et al. (2009) (see also LyndenBell \& Pringle 1974; Hartmann et al. 1998), namely, for each component, the surface density is

$$
\Sigma=\Sigma_{\mathrm{c}}\left(\frac{r}{r_{\mathrm{c}}}\right)^{-\gamma} \exp \left[-\left(\frac{r}{r_{\mathrm{c}}}\right)^{2-\gamma}\right]
$$

The volume density is calculated from the surface density based on vertical hydrostatic equilibrium, using temperatures calculated from a Monte Carlo radiative transfer treatment (Dullemond et al. 2002). The UV radiation field uses the

\footnotetext{
https://www.astro.princeton.edu/ draine/dust/dust.diel.html
} 
Table 2

Parameters for the Disk Structure of TW Hya and DM Tau

\begin{tabular}{|c|c|c|c|c|c|c|c|}
\hline & $\begin{array}{l}M_{\text {dust }} \\
\left(M_{\odot}\right)\end{array}$ & $\begin{array}{c}r_{\text {in }} \\
(\mathrm{au})\end{array}$ & $\begin{array}{l}r_{\text {out }} \\
\text { (au) }\end{array}$ & $\begin{array}{c}r_{\mathrm{c}} \\
(\mathrm{au})\end{array}$ & $\gamma$ & $\begin{array}{l}a_{\min } \\
(\mu \mathrm{m})\end{array}$ & $\begin{array}{l}a_{\max } \\
(\mu \mathrm{m})\end{array}$ \\
\hline \multicolumn{8}{|c|}{ TW Hya } \\
\hline Inner Disk & $1 \times 10^{-9}$ & 0.1 & 3.5 & 5 & 1.0 & 0.9 & 2 \\
\hline Submillimeter Disk & $2 \times 10^{-4}$ & 3.5 & 50 & 40 & 1.5 & $5 \times 10^{-3}$ & $10^{3}$ \\
\hline UV-dominated Disk & $1 \times 10^{-5}$ & 3.5 & 200 & 80 & 1.5 & $5 \times 10^{-3}$ & 1 \\
\hline \multicolumn{8}{|c|}{ DM Tau } \\
\hline Inner Disk & $5 \times 10^{-7}$ & 1 & 4 & 5 & 1.0 & $5 \times 10^{-3}$ & $10^{3}$ \\
\hline Submillimeter Disk & $1 \times 10^{-4}$ & 4 & 100 & 30 & 1.5 & $5 \times 10^{-3}$ & $10^{3}$ \\
\hline UV-dominated Disk & $4 \times 10^{-4}$ & 4 & 500 & 70 & 0.5 & $5 \times 10^{-3}$ & 1 \\
\hline
\end{tabular}

Note. The gas mass is 100 times the total dust mass.

observed stellar spectrum of TW Hya (Herczeg et al. 2002, 2004) as an input and is quantified in units of the wavelength integrated interstellar radiation field (ISRF) $\left(G_{0}=1\right.$ is equivalent to the UV field in the general ISRF; Habing 1968).

The model for DM Tau is similar to TW Hya in that the dust optical constants and size distribution are the same, but the disk physical and chemical parameters are altered based on the observed dust spectral energy distribution, the size and overall distribution of the submillimeter emission disk from the data used here, and the $\mathrm{C}_{2} \mathrm{H}$ molecular emission. For our source specific modeling we did not adjust the intensity of the UV radiation field and we use the TW Hya radiation field for both systems. In general our models show that the primary effects induced by the $\mathrm{UV}$ on the $\mathrm{C}_{2} \mathrm{H}$ abundance occur with a scale much larger than caused by variations of a factor of a few, which is the known difference for these accreting stars (Bergin et al. 2004; Schindhelm et al. 2012; Yang et al. 2012).

The X-ray luminosity for DM Tau is taken to be the preferred value from Henning et al. (2010) of $L_{\mathrm{X}}=3 \times 10^{29} \mathrm{erg} \mathrm{s}^{-1}$, while for TW Hya we adopt $L_{\mathrm{X}}=1.6 \times 10^{30} \mathrm{erg} \mathrm{s}^{-1}$ (Raassen 2009). Our group has explored the cosmic ray ionization rate within disks in general and for TW Hya specifically, suggesting that the rate may be significantly reduced due to interactions with the stellar wind (Cleeves et al. 2013, 2015). Thus we have run our models with a rate that is reduced compared to the interstellar medium by two orders of magnitude $\left(\sim 10^{-19} \mathrm{~s}^{-1}\right.$, with an attenuation parameter of $\left.96 \mathrm{~g} \mathrm{~cm}^{-2}\right)$. Finally, our results are presented for models which have been sampled at $1 \mathrm{Myr}$ for DM Tau; since TW Hya is known to be older we provide results from a model sampled at $5 \mathrm{Myr}$.

Below we use the TW Hya model to explore some generic issues about the origin of $\mathrm{C}_{2} \mathrm{H}$ ring emission; however, it also provides important clues regarding the differing distribution observed in DM Tau. We find that the overall structure of the dust disk is important in making the rings, but it is not enough by itself: some nontrivial chemical effect has to be incorporated to explain the observed morphology and intensity of the rings. In Section 5 we present and discuss more detailed sourcespecific models for TW Hya and DM Tau.

\subsection{Ingredient \#1 for Hydrocarbon Rings: UV-dominated Surface and Outer Disk}

Models of dust evolution generally assume an interstellar grain size distribution as the starting point (e.g., Birnstiel et al. 2012). The settling, coagulation, fragmentation, and radial drift of dust grains occur on timescales that depend on turbulence and the overall gas mass distribution of the disk. The fact that the dust disk seen in submillimeter emission (Figure 2) is smaller than the gas-phase hydrocarbons and $\mathrm{CO}$ emission suggests that millimeter-sized (or larger) grains have undergone radial drift in both TW Hya and DM Tau (see also the discussion by Andrews 2015). These heavy grains are difficult for turbulence to loft to high altitude (Dullemond \& Dominik 2004), which is supported by detailed studies of the dust emission for TW Hya (Andrews et al. 2012). Thus, the larger grains are radially and vertically stratified.

Smaller grains are present since both systems show scattered near-IR light that extends beyond the disk traced by submillimeter emission (Weinberger et al. 2002; Schneider et al. 2005). Both disks have therefore evolved to the point where there is significant stratification in the dust mass. To explore the effects of this solid mass stratification on the chemistry, we constructed two models: the first is the threecomponent model (inner disk, submillimeter disk, UVdominated disk) as in Table 2 (similar to the eventual TW Hya model) which has $90 \%$ of the dust mass confined within $50 \mathrm{au}$. The remainder of the dust mass is in smaller grains distributed over the whole disk. This model therefore has a radial break in the dust distribution. The second model assumes that all the dust mass is present as smaller grains that are smoothly distributed throughout the disk.

Figure 5 shows the density structure, the UV field (the $G_{0}$ factor), and the $\mathrm{C}_{2} \mathrm{H}$ density for a disk with and without dust mass stratification. In the top panels, since UV-absorbing grains are present throughout the disk, the radiation field is strongest at the disk surface, and decreases with depth toward the disk midplane. In the case where most of the dust mass is confined, the overall density structure shows some differences, but the largest change is seen in the propagation of UV radiation. Here the UV photons traverse the entire disk surface, wrapping around the millimeter disk, and are even present in the outer disk midplane $\left(G_{0} \gtrsim 1\right)$. In this template model (motivated by TW Hya) the dust mass difference between the millimeter-disk and the overall disk is $\sim 10$. Larger grains ( $>$ millimeter-sized) have reduced ability to absorb UV radiation, with a factor of $\sim 20$ reduction in the (mass) absorption coefficient at $1200 \AA$ between a $0.1 \mu \mathrm{m}$ grain and a larger millimeter-sized pebble (based on the optical constants from Draine \& Lee 1984). The significant difference in mass and density between the large and small grain population in the stratified model overcomes this imbalance; it is the disk traced 

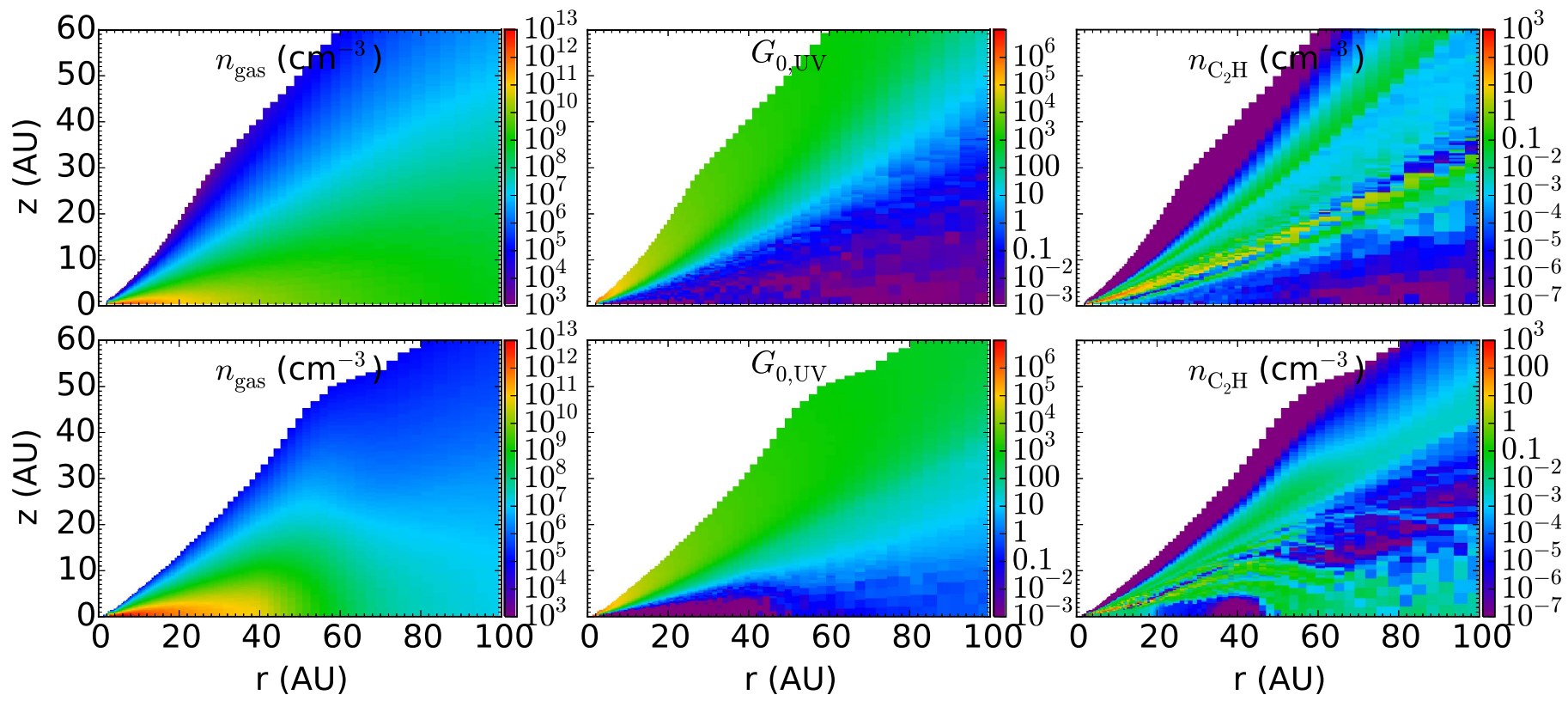

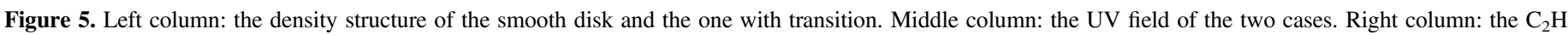
density distribution of the two cases.

by millimeter-grains that is opaque to UV photons. In the upper layers and outer disk, the gas is essentially a dense PDR (Tielens \& Hollenbach 1985). For DM Tau, the UV dominated disk has more dust mass than the disk traced by submillimeter emission (Table 2), but this is spread over a significantly larger volume. In the following we will label this region the UVdominated disk.

In the dense interstellar medium the reactive $\mathrm{C}_{2} \mathrm{H}$ radical has been characterized as a tracer of transition zones within PDRs where the gas is rich in atoms and simple molecules with short reactive lifetimes (see Nagy et al. 2015, for a recent study). Thus in Figure 5 there is a dramatic difference in the overall $\mathrm{C}_{2} \mathrm{H}$ distribution. In the smooth model $\mathrm{C}_{2} \mathrm{H}$ is mostly confined to the upper layers of the disk where the UV field exceeds $G_{0}>1$. In this model the primary formation pathways for $\mathrm{C}_{2} \mathrm{H}$ are related to the overall $\mathrm{C} / \mathrm{O}$ ratio in the disk, i.e., the presence of atomic carbon. The key formation pathways are:

$$
\begin{aligned}
& \mathrm{C}+\mathrm{H}_{3}^{+} \rightarrow \mathrm{CH}_{3}^{+}, \\
& \mathrm{CH}_{3}^{+}+\mathrm{H}_{2} \rightarrow \mathrm{CH}_{5}^{+}, \\
& \mathrm{CH}_{5}^{+}+\mathrm{H}_{2} \rightarrow \mathrm{CH}_{3}+\mathrm{H}_{2}, \\
& \mathrm{CH}_{3}+\mathrm{C} \rightarrow \mathrm{C}_{2} \mathrm{H}_{2}, \\
& \mathrm{C}_{2} \mathrm{H}_{2}+h \nu \rightarrow \mathrm{C}_{2} \mathrm{H}+\mathrm{H}, \\
& \mathrm{C}_{2} \mathrm{H}_{2}+\mathrm{H}_{3}^{+} \rightarrow \mathrm{C}_{2} \mathrm{H}_{3}^{+}+\mathrm{H}_{2}, \\
& \mathrm{C}_{2} \mathrm{H}_{3}^{+}+\mathrm{e}^{-} \rightarrow \mathrm{C}_{2} \mathrm{H}+\mathrm{H}_{2} .
\end{aligned}
$$

We note that this list is not comprehensive and other reactions can play a role. However, the critical link is the need for some atomic carbon to be extracted from $\mathrm{CO}$ via photodissociation. Correspondingly, there is some structure in the $\mathrm{C}_{2} \mathrm{H}$ abundance. The top layer traces the $\mathrm{CO}$ self-shielding zone and the lower vertical layer is the result of carbon extraction from $\mathrm{CO}$ via reactions with $\mathrm{He}^{+}$, which is the product of $\mathrm{He} \mathrm{X}$-ray ionization.

In the model with dust mass stratification, the lower optical depth for UV photons activates such chemistry over a greater disk volume. Thus $\mathrm{C}_{2} \mathrm{H}$ is observed to be present at high levels even in the outer disk midplane; the magnitude of the change is $>1000$ in abundance. It is this difference that is one of the key pieces for the origin of the outer $\mathrm{C}_{2} \mathrm{H}$ ring in both TW Hya and DM Tau. Figure 6 shows resulting images of the $\mathrm{C}_{2} \mathrm{H}$ emission in the $N=4-3$ transition. As can be seen, in the model with dust mass stratification, the $\mathrm{C}_{2} \mathrm{H}$ emission exhibits a ring at $\sim 50 \mathrm{au}$, close to the rim of the inner dust disk traced observationally by submillimeter emission. It is near the outer radius of the pebble-dominated disk where the UV photons have their greatest effect-as they can influence material closer to the midplane and extend the influence of the PDR over a greater column. In this case pebbles refers to millimeter and $\mathrm{cm}$ sized-grains. Thus, the smooth model does not show sharp features like this and dust evolution is expected to have a significant impact on the observed distribution of hydrocarbons (see also Semenov \& Wiebe 2011).

The emission distribution in the right-hand panel of Figure 6 is somewhat similar to that of DM Tau; although on a smaller physical scale. The overall emission is also much weaker as this model is over an order of magnitude weaker than the $\mathrm{C}_{2} \mathrm{H}$ ring emission in both sources. An additional physical/chemical mechanism must also be operative to produce the central hydrocarbon emission hole detected in TW Hya.

\subsection{Ingredient \#2 for Hydrocarbon rings: Depletion of Oxygen and Carbon, and Variable C/O Ratio}

Dust redistribution alone does not complete the picture as the stratified model does not match the level of observed emission; nor does it have an inner hole. There are a few possibilities to raise the emission of $\mathrm{C}_{2} \mathrm{H}$ in the ring. First, there could be an order of magnitude more gas in the outer disk than included in our models. But our models already assume a large gas mass of $\sim 0.02-0.04 M_{\odot}$. In addition, our template model is based on the Du et al. (2015) exploration of TW Hya and a higher outer disk gas mass will raise the HD emission to higher levels than observed (Bergin et al. 2013). Based on HD, DM Tau has a 

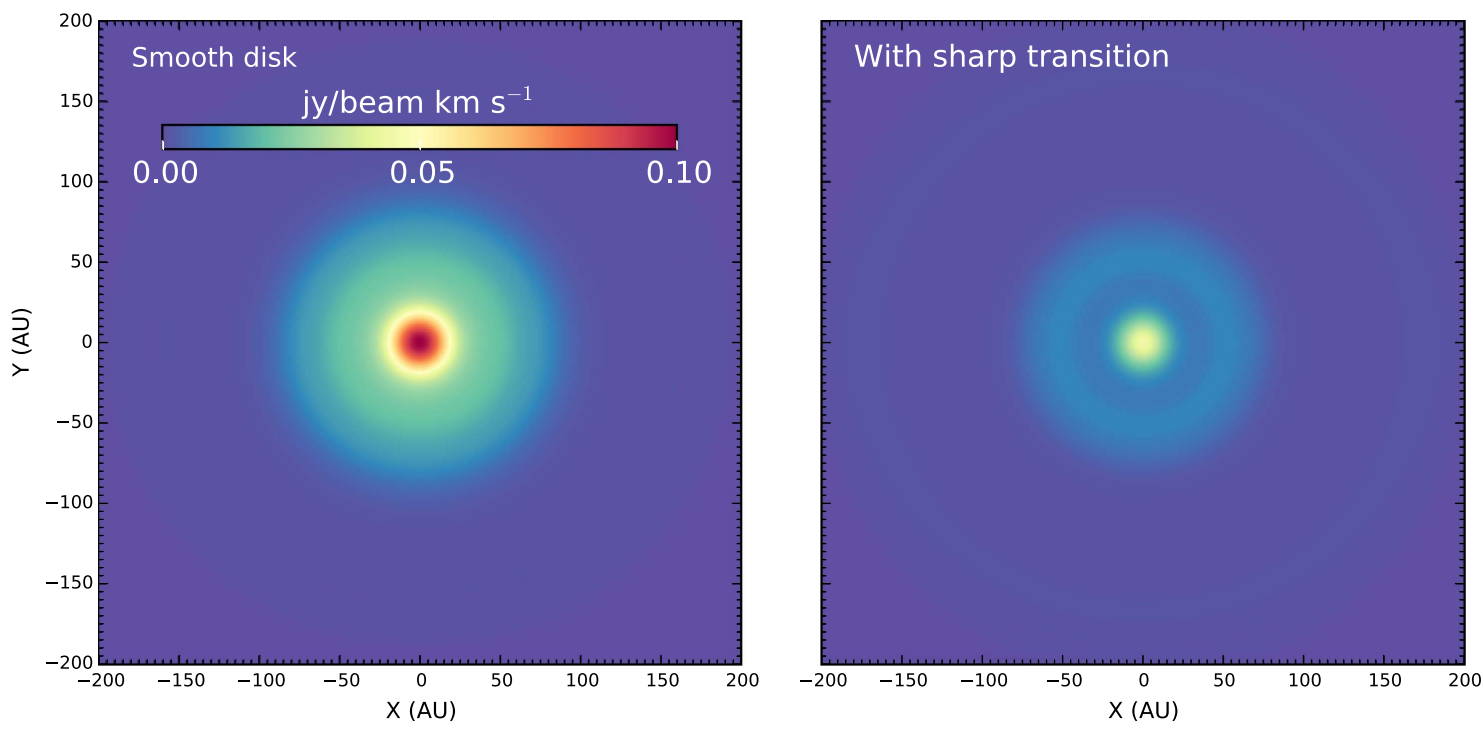

Figure 6. $\mathrm{C}_{2} \mathrm{H} J=7 / 2-5 / 2, F=4-3$ and $3-2$ emission from a disk with smooth density profile (left panel) and from a disk with a transition in column density (right); see Figure 5 for the two disk structures. The images have been convolved with a Gaussian beam with full width half maximum of 0 !. 7 . The two panels share the same color scale.

Table 3

Initial Abundance Parameters for Variable C/O Ratio

\begin{tabular}{lccc}
\hline \hline Species & $\mathrm{C} / \mathrm{O}=0.4$ & $\mathrm{C} / \mathrm{O}=1$ & $\mathrm{C} / \mathrm{O}=2$ \\
\hline $\mathrm{C}$ & 0 & $1.4 \times 10^{-4}$ & $1.4 \times 10^{-4}$ \\
$\mathrm{CO}$ & $1.4 \times 10^{-4}$ & 0 & 0 \\
$\mathrm{H}_{2} \mathrm{O}_{\text {ice }}$ & $1.8 \times 10^{-4}$ & $1.4 \times 10^{-4}$ & $0.7 \times 10^{-4}$ \\
\hline
\end{tabular}

Note. Abundances do not reflect the total $\mathrm{C} / \mathrm{O}$ ratio as they do not include refractory carriers.

comparable mass to that of TW Hya (McClure et al. 2016). Thus it is difficult for mass to be a primary variable both within and between a given source. The fall off of mass with radius is also constrained by the radial distribution of small grains (e.g., Andrews 2015). Second, we could change the strength of the UV field, but again our models are constrained by the observed radiation fields of both sources, along with the modeled dust emission (Herczeg et al. 2002; Bergin et al. 2004). Finally, the gas temperature could play a role, but is constrained by the emission from CO and its isotopologues (Thi et al. 2010; Du et al. 2015).

We propose instead an alternative: that the dust mass stratification also implies a stratification in the volatile inventory of the disk. Specifically, as the dust grains settle and drift, they also carry their ice mantles into the inner disk, leaving the upper layers of the outer disk depleted in volatile elements. In the case of TW Hya and DM Tau this is supported by the weak emission from ground state transitions of water vapor (Bergin et al. 2010; Hogerheijde et al. 2011; Du et al. 2015) along with the inferred depletion of carbon in TW Hya (Favre et al. 2013; Tsukagoshi et al. 2015; Kama et al. 2016a, 2016b). Several papers have explored various aspects of this issue (Bergin et al. 2014; Furuya \& Aikawa 2014; Du et al. 2015; Reboussin et al. 2015). A key point is that, due to its reduced volatility, water will be more susceptible to midplane sequestration than species such as $\mathrm{CO}$. Thus the upper layers of the outer disk will have $\mathrm{C} / \mathrm{O} \gtrsim 1$ due to settling and drift. This

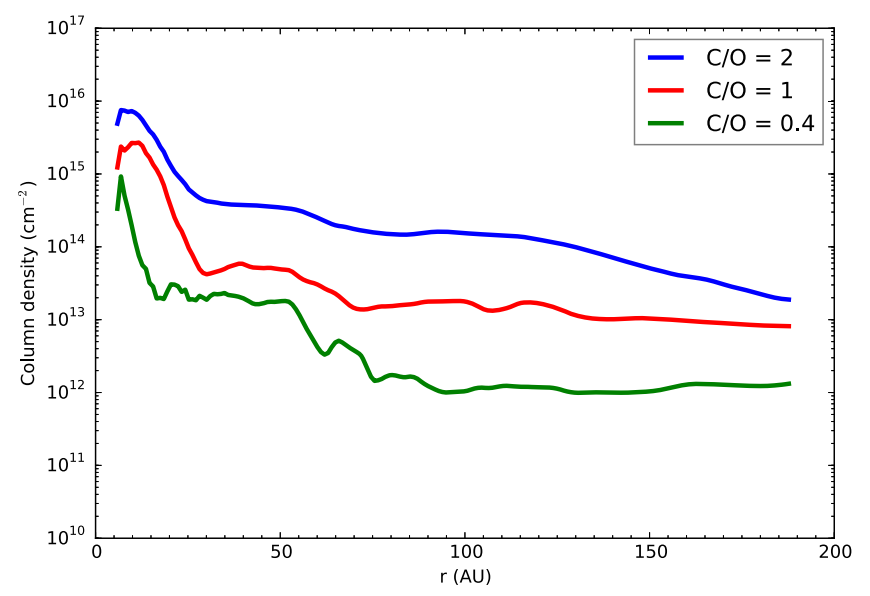

Figure 7. Variation of the $\mathrm{C}_{2} \mathrm{H}$ column density in the fiducial three-component model of TW Hya (described in Section 4) for different (uniform) C/O elemental abundance ratio.

volatile stratification leaves $\mathrm{C}$ bearing species in a sea of $\mathrm{H}_{2}$ with abundant ionization. As predicted by Du et al. (2015) this gas would be a hydrocarbon (and potentially also hydronitrogen) factory. Indeed, in TW Hya, as shown in Figures 1 and 4, the $\mathrm{C}_{2} \mathrm{H}$ and $c-\mathrm{C}_{3} \mathrm{H}_{2}$ follow a common spatial distribution.

Here we will use our template model from Section 4.1 to explore the general effects of the relative carbon to oxygen depletion on $\mathrm{C}_{2} \mathrm{H}$, changing the overall $\mathrm{C} / \mathrm{O}$ ratio uniformly in the entire disk. In Table 3 we provide initial abundances of relevant species for different uniform $\mathrm{C} / \mathrm{O}$ ratios in the disk. Generally, and based on years of study of the interstellar medium, it is believed that the main carbon carrier is $\mathrm{CO}$ and carbonaceous grains, while the main carriers of oxygen are water ice and silicate grains, although other species contribute (e.g., $\mathrm{CO}_{2}$; Whittet 2010). In the models below we ignore the refractory contributions and reduce the water ice abundance to mimic a changing $\mathrm{C} / \mathrm{O}$ ratio. When the $\mathrm{C} / \mathrm{O}$ ratio exceeds unity we must also redistribute some of the carbon out of $\mathrm{CO}$ 

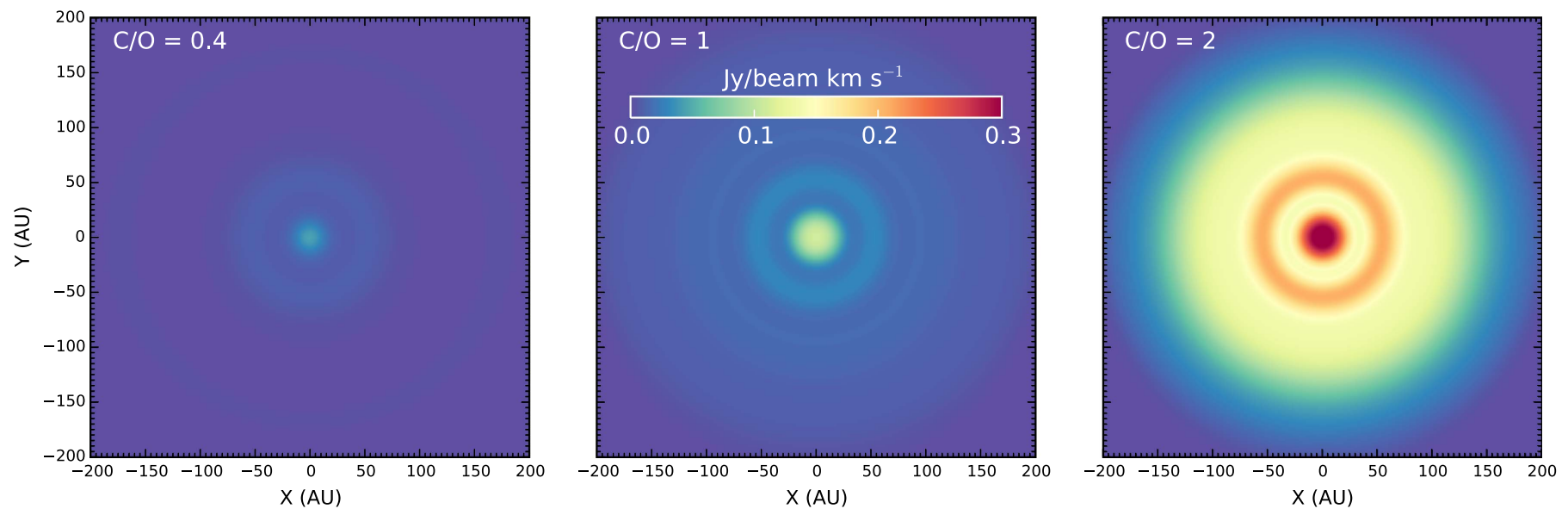

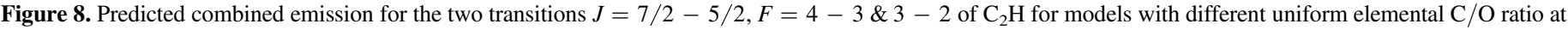
the same integrated flux color stretch. For this model we use the three component TW Hya model described in Section 4.

into other carriers and in this instance we place the carbon in neutral atomic form.

The results from these models in terms of implications for the radial column density variation of $\mathrm{C}_{2} \mathrm{H}$ are shown in Figure 7. As one removes oxygen from the chemistry the $\mathrm{C}_{2} \mathrm{H}$ column density increases, with a larger (one order of magnitude) increase beyond $\sim 75 \mathrm{au}$. The key issue here is the role oxygen plays in the destruction of carbon-bearing species that are not as stable as $\mathrm{CO}$ or $\mathrm{CO}_{2}$. In the case of the radical $\mathrm{C}_{2} \mathrm{H}$ the main destruction paths are as follows:

$$
\begin{aligned}
\mathrm{O}+\mathrm{C}_{2} \mathrm{H} & \rightarrow \mathrm{CO}+\mathrm{CH}, \\
\mathrm{N}+\mathrm{C}_{2} \mathrm{H} & \rightarrow \mathrm{C}_{2} \mathrm{~N}+\mathrm{H}, \\
\mathrm{C}+\mathrm{C}_{2} \mathrm{H} & \rightarrow \mathrm{C}_{3}+\mathrm{H}, \\
\mathrm{C}^{+}+\mathrm{C}_{2} \mathrm{H} & \rightarrow \mathrm{C}_{3}^{+}+\mathrm{H} .
\end{aligned}
$$

Atomic oxygen is present in the model due to photodesorption of water ice and its subsequent photodissociation (Hollenbach et al. 2009). But if oxygen is sequestered on larger grains that settle to the midplane and drift inwards, then the oxygen abundance of the upper layers and of the outer disk will be reduced. The lack of oxygen removes a key species that is keeping the hydrocarbon chemistry in check, but also elevates abundances of other important species such as $\mathrm{N}, \mathrm{C}$, and $\mathrm{C}^{+}$. This process elevates the abundance of hydrocarbons as discussed by $\mathrm{Du}$ et al. (2015). Modest removal of oxygen produces relatively large enhancements in the $\mathrm{C}_{2} \mathrm{H}$ column density across all radii.

In Figure 8 we show the resulting emission profiles for models with different $\mathrm{C} / \mathrm{O}$ ratios. These simulated images show some structure, but here we focus solely on the overall intensity. The underlying physical model was originally developed for TW Hya, so one can compare with the panel for the $J=7 / 2-5 / 2, \quad F=4-3$ and $3-2$ combined transitions in Figure 1. As can be seen for the model with a "normal" abundance of carbon and oxygen $(\mathrm{C} / \mathrm{O}=0.4)$ the emission is well below observed values. A $\mathrm{C} / \mathrm{O}$ ratio of unity comes closer, but $\mathrm{C} / \mathrm{O} \gtrsim 1$ is needed (in this model) to approach observed values. These models roughly match the distribution seen in DM Tau; however, this model is at a much smaller physical scale. Nonetheless it does hint that this process could be ongoing in that source.
The uniformly increased $\mathrm{C} / \mathrm{O}$ ratio does not explain the inner hole of the $\mathrm{C}_{2} \mathrm{H}$ emission for TW Hya. There are two possible ways to create the inner hole. The first is to enhance the oxygen abundance relative to carbon in the inner disk, which is exactly the opposite of the outer disk. The spatial $\mathrm{C} / \mathrm{O}$ ratio can be influenced by the various snow lines, which is discussed in detail by Öberg et al. (2011b). The size of the inner hole is $\sim 20 \mathrm{au}$, which lies inside the surface CO snowline of TW Hya at 25-30 au (Qi et al. 2013). When CO ice sublimates it can influence hydrocarbon chemistry. However, this is a two way street in the sense that when dissociated, $\mathrm{CO}$ provides an oxygen atom that readily destroys $\mathrm{C}_{2} \mathrm{H}$ (going back to $\mathrm{CO}$ ), it also leaves a reactive $\mathrm{C}^{+}$ion in the gas, which can produce hydrocarbons (e.g., Bergin et al. 2014). The $\mathrm{H}_{2} \mathrm{O}$ sublimation front has been studied by Blevins et al. (2016) and are inferred to exist within the inner few au of the disk surface for $\mathrm{T}$ Tauri disk systems. $\mathrm{CO}_{2}$ is more difficult to study but is known to be present in ices (Öberg et al. 2011a) and comets (Mumma \& Charnley 2011); based on estimated bond strengths it should evaporate between the snow-lines of water and $\mathrm{CO}$ (Martín-Doménech et al. 2014) in between 5-10 au. The injection of $\mathrm{H}_{2} \mathrm{O}$ and/or $\mathrm{CO}_{2}$ will lead to a minimum hole size in the $\mathrm{C}_{2} \mathrm{H}$ abundance.

However, although the enhancement of oxygen relative to carbon could create an inner hole, this leads to an inconsistency with observations if carbon itself is at the ISM abundance. It is known from observations of $\mathrm{CO}$ isotopologues that the $\mathrm{CO}$ abundance is reduced relative to the canonical ISM value of $10^{-4}$ by nearly two orders of magnitude (Favre et al. 2013; Nomura et al. 2016; Schwarz et al. 2016). This forces us to the second alternative that the abundance of chemically active carbon must be reduced in the inner $\sim 25$ au, while the behavior of oxygen in the inner disk is unclear. The strong water emission in TW Hya (Zhang et al. 2013) shows that there is some sublimation within the inner $10 \mathrm{au}$. It is also plausible that some, potentially large, fraction of the carbon atoms are assimilated into refractory organic grains and do not return to the gas phase even in the inner warm region.

In summary, the settling and migration of icy grains reduces the UV opacity in the surface and the outer disk. At the same time, the grains initially carry greater amounts of water than carbon-bearing species, leading to a $\mathrm{C} / \mathrm{O}$ ratio of at least 1 in the upper layers of the outer disk. A variety of mechanisms 

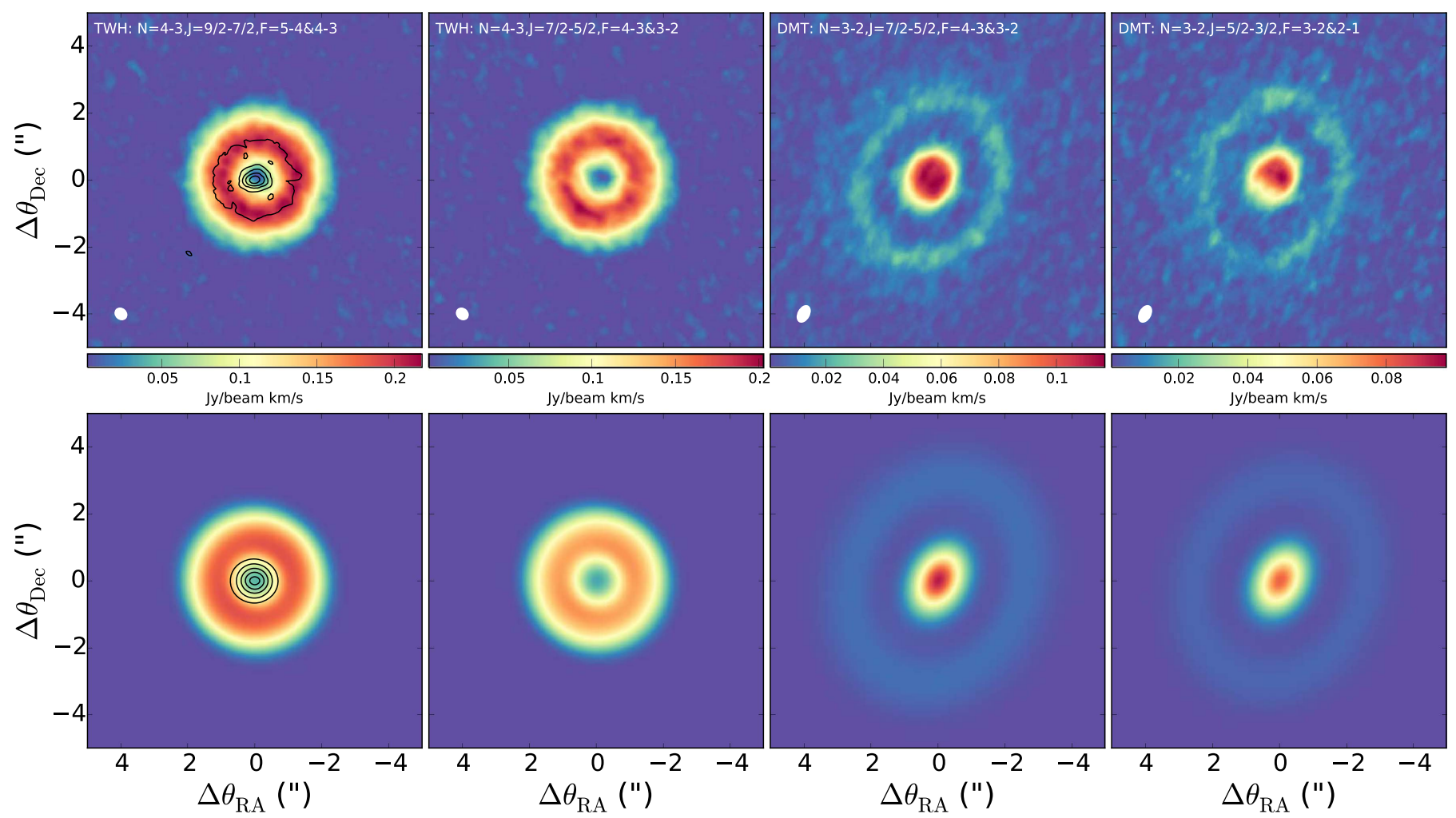

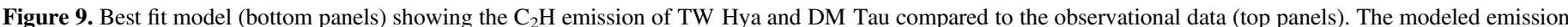

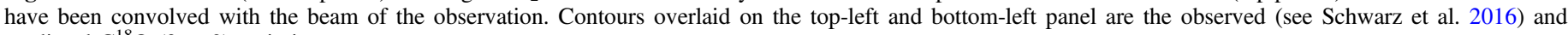
predicted $\mathrm{C}^{18} \mathrm{O}(3-2)$ emission.

have been explored in the literature that liberate carbon from CO (Bergin et al. 2014; Furuya \& Aikawa 2014; Du et al. 2015; Reboussin et al. 2015), which can enhance the $\mathrm{C} / \mathrm{O}$ ratio to higher levels and increase the abundance of $\mathrm{C}_{2} \mathrm{H}$ and other hydrocarbons. Over time this can also deplete the inner disk of carbon. When combined with sublimation closer to the star a deep central hole can develop over time.

\section{DISCUSSION}

There are a number of issues unresolved. TW Hya and DM Tau disks have very different observed emission morphologies. DM Tau has stronger central emission, though its central region seems to be a resolved hole or flattening (see Figure 3), with a much more extended, weaker, outer ring. The DM Tau disk is substantially larger in size, although the gas masses of the two are comparable (McClure et al. 2016). Furthermore, we have assumed that $\mathrm{CO}$ is the source of carbon for $\mathrm{C}_{2} \mathrm{H}$, but the photodegradation of aliphatic or aromatic hydrocarbon grains could also be a source term for the needed carbon (see discussion in Kastner et al. 2015). In the following we present source specific models to match the emission for both sources, explore the excitation characteristics of $\mathrm{C}_{2} \mathrm{H}$ emission, and discuss additional sources of carbon for the production of the observed species.

\subsection{Source Specific Models}

In our list of key ingredients to produce the hydrocarbon ring emission we illustrated the increased penetration of UV radiation due the combined effects of settling and radial drift and showed how $\mathrm{C}_{2} \mathrm{H}$ emission can be boosted by global changes in the $\mathrm{C} / \mathrm{O}$ ratio. To link these physical and chemical
Table 4

Depletion parameters for oxygen and carbon in TW Hya and DM Tau, as defined in Equations (7)-(9)

\begin{tabular}{lccll}
\hline \hline & $\begin{array}{c}r_{0} \\
(\mathrm{au})\end{array}$ & $\begin{array}{c}r_{\mathrm{s}} \\
(\mathrm{au})\end{array}$ & $a$ & $b$ \\
\hline TW Hya (O) & 15 & 3 & 0.9 & 0.6 \\
TW Hya (C) & 60 & 5 & 0.7 & 0.2 \\
DM Tau (O) & 70 & 20 & 1 & 0.2 \\
DM Tau (C) & 90 & 20 & 0.9 & 0.6 \\
\hline
\end{tabular}

effects together, the distribution of key carriers in the elemental abundance pools must be non-uniform. The ice mass likely follows the dust mass; hence the relative distribution of the volatiles left in the gas must reflect this in some fashion. Furthermore there might be some reset process interior to the snow lines of key carriers, though this is not guaranteed to happen for two reasons. First, some fraction of the ice may be sequestered into large $\mathrm{km}$-sized planetesimals and will not evaporate at the traditional snow-line (Piso et al. 2015). Second, if CO processing is very efficient such that the oxygen from $\mathrm{CO}$ is incorporated into water and the carbon into larger species (e.g., Bergin et al. 2014), then the CO abundance would be depleted in the gas and at the surface $\mathrm{CO}$ snow line. Thus on crossing the snow line there will be net loss of oxygen and carbon in the gas phase, the exact amount of which depends on the timescales for chemical processing.

In the Appendix we provide the parameterization we adopt to model the uneven distribution of elements. This parameterization is somewhat arbitrary and more realistic chemical 

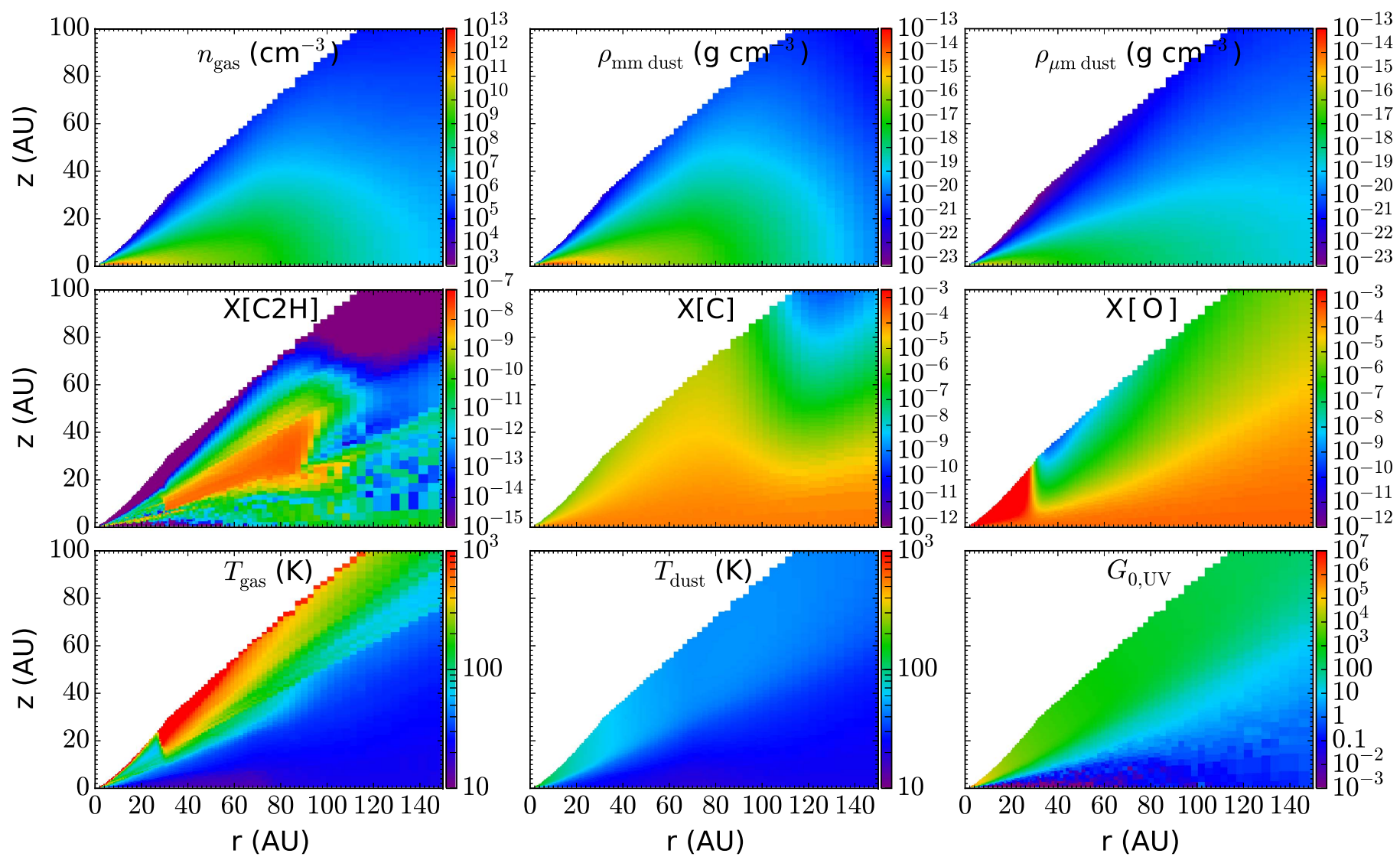

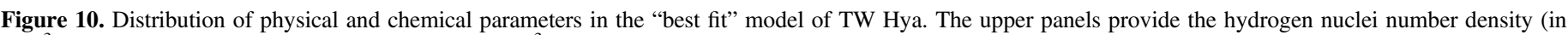

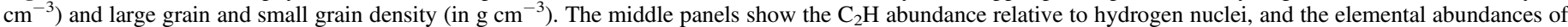
carbon and oxygen. The bottom panels show the calculated gas and dust temperatures followed by the UV radiation field in units of $G_{0}$.

models need to be constructed that include the formation of bodies large enough to stop drift and halt the formation of small grains via collisions that can be lofted to the warmer UVexposed surface, where volatiles can be released from the ice mantles. However, our parameterized model captures key aspects of the proposed picture where volatiles are sequestered in the midplane and, due to drift, reside predominantly as ices in the inner tens of au of the disk.

The "best fit" emission images of $\mathrm{C}_{2} \mathrm{H}$ for both disks are shown in Figure 9. The parameters describing the basic disk structure are shown in Table 2 and the corresponding parameters for the elemental abundance distribution of carbon and oxygen are provided in Table 4 for TW Hya and DM Tau, the meanings of which are defined in Appendix A.

\subsubsection{TW Hya}

In Figure 10 we show the distribution of physical parameters for the "best fit" model for the distribution of $\mathrm{C}_{2} \mathrm{H}$ in TW Hya. For reference we show the contribution function for this emission map in Appendix B (Figure 15). In this model there is one central facet and two areas of uncertainty. The central requirement is that in the ring, the $\mathrm{C} / \mathrm{O}$ ratio is $\gtrsim 1$ to reproduce the observed intensity. $\mathrm{C}_{2} \mathrm{H}$ is formed in the UV-dominated disk in the upper layers and down to the midplane wrapping around the inner disk comprised of larger grains. The two areas of uncertainty regard: (1) the central hole in the emission and (2) the outer radius of the emission ring, which is beyond primary edge of the pebble disk but interior to the outer radius of ${ }^{12} \mathrm{CO}$ emission. With our assumptions discussed below, the predicted emission distribution shown in Figure 9 matches observations within a factor of 1.5 .

For the inner edge, in our models the elemental carbon abundance is reduced in the upper emissive layers within 20 au. This is required by both the CO isotopologue data (Favre et al. 2013; Nomura et al. 2016; Schwarz et al. 2016) and the $\mathrm{C}_{2} \mathrm{H}$ data. One possible reason for this may be an uneven distribution of pebbles in the disk. Tsukagoshi et al. (2016) find that the TW Hya millimeter-wave (138 and $230 \mathrm{GHz})$ dust spectral index increases from the inner edge until $\sim 20 \mathrm{au}$. This is generally interpreted as due to grain growth in regions with lower values of the spectral index (e.g., Pérez et al. 2015). Thus perhaps there is an excess of larger $\mathrm{cm}$-sized or larger grains that incorporate carbon in more refractory form giving rise to the central depletion. The modeled $\mathrm{C}^{18} \mathrm{O}$ emission also matches the result of Schwarz et al. (2016), which is overlaid on the top/bottom left panels of Figure 9. A normal ISM abundance of carbon would over-predict the $\mathrm{C}^{18} \mathrm{O}$ emission, and would not be able to reproduce the high contrast (i.e., the ratio between the intensity in the ring to the intensity in the central hole) of the $\mathrm{C}_{2} \mathrm{H}$ ring.

The second issue then regards the physics/chemistry dictating the position of the outer ring edge. From observations of ${ }^{13} \mathrm{CO}$ in the TW Hya disk (Nomura et al. 2016; Schwarz et al. 2016) the emission intensity exhibits a sharp decline near $60-70$ au $\left(\sim 1\right.$ !'2) at a point where the $\mathrm{C}_{2} \mathrm{H}$ emission also begins to decline. Thus, one possibility is that the edge of the emission ring is set by an overall decrease in the gas surface 


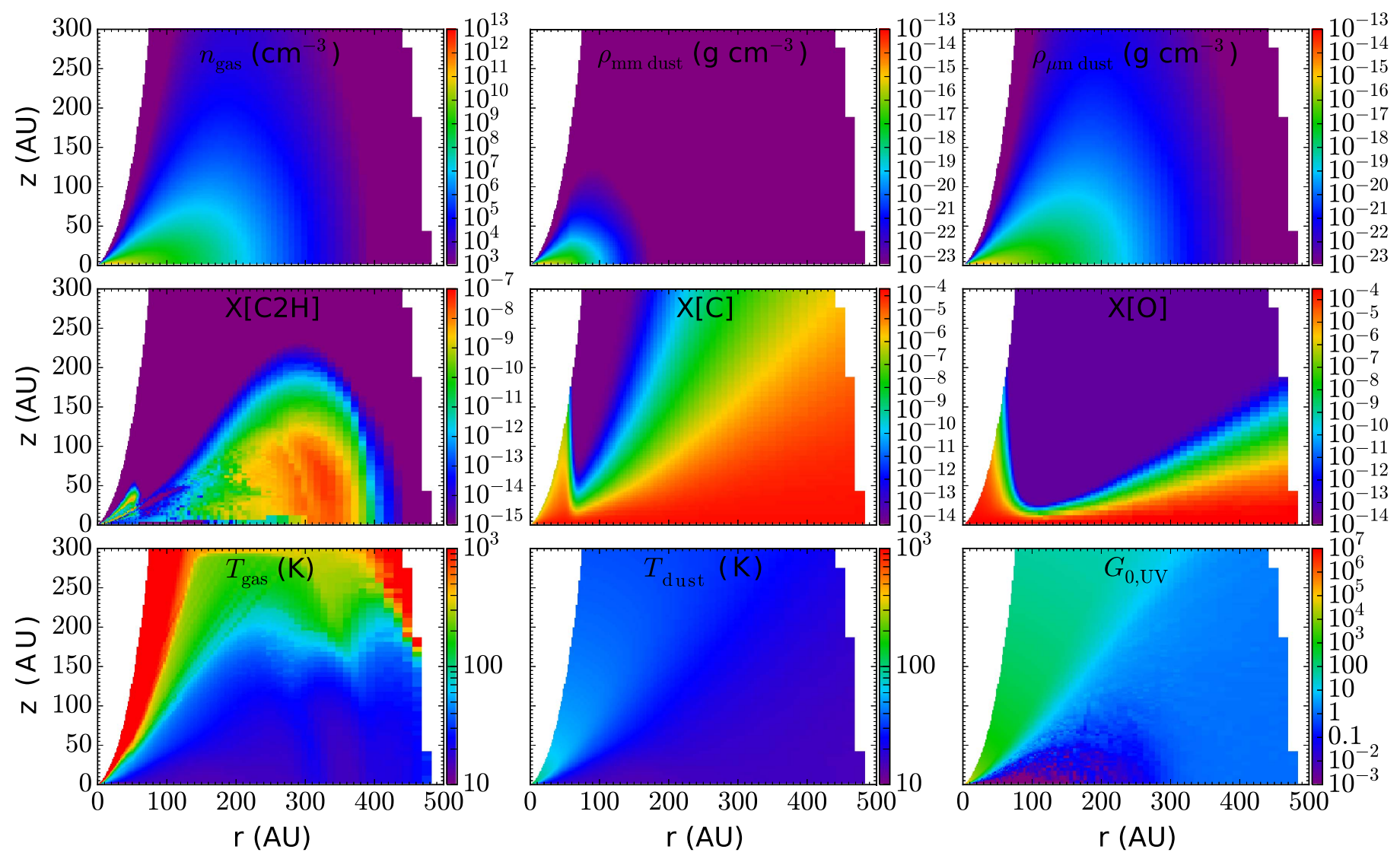

Figure 11. Same as Figure 10, but for DM Tau.

density. Of course ${ }^{12} \mathrm{CO}$ and scattered star light are observed outside of $\sim 2^{\prime \prime}$ (Andrews et al. 2012; Debes et al. 2013), but this could be due to a more tenuous outer disk. Alternately, the outer edge could also be set by carbon depletion (which is the case in the parameterization of Table 4) and the hydrocarbon emission rings are just maxima where carbon exists in the gaseous state. If the temperature of the young TW Hya disk was below the $\mathrm{CO}$ sublimation temperature $(\sim 20 \mathrm{~K})$ grains that grew in the midplane at large distances and drifted inwards would "steal" carbon from the outer disk leaving a carbon depleted warm molecular layer on the surface as the carbon repository. Thus if any part of the disk will suffer strongly from these effects it must be the outer disk where the combined effects of settling and drift deplete this region of icy grains. Since drift appears to be a general result, we would predict similar chemical effects in all disks where evidence of drift is observed.

In our models we have made an assumption of a constant gas/dust mass ratio throughout the disk, regardless of our assumptions regarding the grain mass distribution. Consequently, in our generic model where the grain mass is concentrated in the inner tens of au and in the midplane, the gas is also centrally concentrated and simultaneously depleted in the outer disk. The best estimate we have on the gas mass comes from HD, which is a better probe of the mass of the inner disk ( $<40$ au; Bergin et al. 2013). Therefore, the gas mass of the outer disk is less well constrained. High resolution studies of CO isotopologues toward TW Hya with ALMA find that that the ${ }^{13} \mathrm{CO}$ emission begins to sharply drop beyond 60 au to below detectable levels (Nomura et al. 2016; Schwarz et al. 2016). This drop might hint at a change in the outer disk gas mass (or to the carbon abundance). Thus, it is fair to question whether the reproduction of the $\mathrm{C}_{2} \mathrm{H}$ emission requires the a priori variation in the $\mathrm{C} / \mathrm{O}$ ratio to boost its abundance, as the mass could be higher in the outer disk than what is currently in our models.

To check the effect of the outer disk gas mass, we have run a model with a factor of 10 higher gas mass. We find that the resulting $\mathrm{C}^{18} \mathrm{O}$ emission is significantly elevated over observed levels, while the $\mathrm{C}_{2} \mathrm{H}$ emission is still well below observed values. To reproduce the observed intensities in this higher mass model, we require carbon depletion and $\mathrm{C} / \mathrm{O}>1$. There are additional constraints for different disk gas models such as Kama et al. (2016b) who explore the abundance of $\mathrm{C}_{2} \mathrm{H}$ (and other carbon bearing atoms/molecules) in TW Hya assuming a gas/dust ratio of 200. They find a similar result and require carbon depletion and $\mathrm{C} / \mathrm{O}>1$. Thus the high $\mathrm{C} / \mathrm{O}$ required to explain the $\mathrm{C}_{2} \mathrm{H}$ emission appears to be robust.

\subsubsection{DM Tau}

For DM Tau, the comparison of our best fit model emission predictions to observations is shown in Figure 9. As shown in Figure 3 and discussed in Section 3, DM Tau has two $\mathrm{C}_{2} \mathrm{H}$ rings. The inner ring is at $R \sim 50$ au and the outer weaker ring is observed at $R \sim 350$ au. Figure 11 shows distribution of physical and chemical parameters from our model that best matches the DM Tau emission distribution, which is compared to observations in Figure 9. As in TW Hya the outer $\mathrm{C}_{2} \mathrm{H}$ ring appears at the edge of the pebble disk traced by $1.3 \mathrm{~mm}$ 


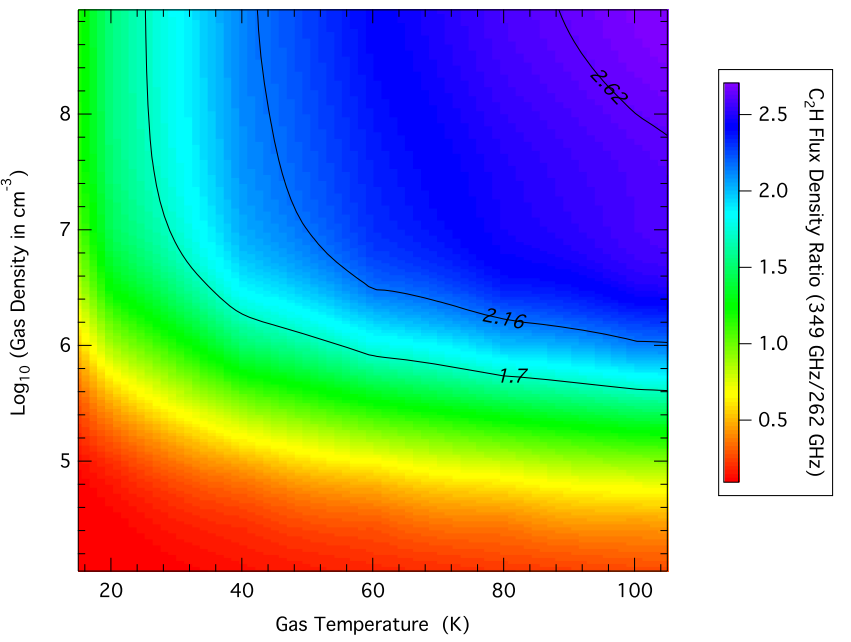

Figure 12. Excitation calculations for the flux density ratio of the $N=4-3$, $J=\frac{9}{2}-\frac{7}{2}, F=4-3$ to $N=3-2, J=\frac{9}{2}-\frac{7}{2}, F=4-3$ transitions. The observed ratio (2.16) with $1 \sigma$ error bars is shown as contours

emission as there is fainter $(3 \sigma)$ extended emission seen in our $1.3 \mathrm{~mm}$ image out to $300 \mathrm{au}$. It is this point where the UVdominated disk can begin to penetrate to greater depth and enhance the column of UV-exposed gas and dust. Our models have some difficulty matching the sharpness of the outer disk ring, though the observational data is limited by the noise level in the outer disk.

As noted in Section 3.3 the inner $\mathrm{C}_{2} \mathrm{H}$ emission ring observed in DM Tau could be due to either dust optical depth or perhaps the beginning of carbon depletion in the inner disk such as seen in TW Hya. Due to this uncertainty we did not model this central hole in greater detail. However, the inner disk still has strong $\mathrm{C}_{2} \mathrm{H}$ emission, which is clearly distinct from TW Hya. An additional notable difference is that DM Tau has a larger abundance of elemental carbon present in the gaseous state. Based on HD measurements TW Hya and DM Tau have roughly comparable gas masses (McClure et al. 2016). However, the $C O$ mass derived for DM Tau from $C^{18} \mathrm{O}$ observations is a factor of 18 higher than TW Hya (Williams \& Best 2014). Without significant carbon depletion, our models (e.g., Figure 6) predict strong central emission consistent with observations. Thus, we interpret the strength of the central emission in DM Tau as a likely time-dependent effect. The combined effects of settling and radial drift will have a stronger influence on the outer disk. The lower density outer disk will also be more transparent to UV radiation than the denser inner disk. Hence the effects of carbon extraction from $\mathrm{CO}$ would have greater efficiency in the outer disk. Over time these effects will become more significant in the inner disk until the system evolves toward the central carbon depletion as seen in TW Hya.

\subsection{Excitation Analysis}

From our modeling of TW Hya (Figures 9 and 10) and the contribution function (Figure 15) we predict that $\mathrm{C}_{2} \mathrm{H}$ is emitting from layers with $\mathrm{n}_{\mathrm{H}_{2}} \sim 10^{7}-10^{8} \mathrm{~cm}^{-3}$ in the warm $\left(T_{\text {gas }} \sim 40 \mathrm{~K}\right)$ surface layers, with additional contribution from a denser midplane. Kastner et al. (2015) used an analysis of the $N=4-3 / N=3-2$ ratio to constrain the excitation conditions of the $\mathrm{C}_{2} \mathrm{H}$ emission. Based on their analysis the emission originates from relatively warm $(T>40 \mathrm{~K})$ but lower density, $n_{\mathrm{H}_{2}} \ll 10^{7} \mathrm{~cm}^{-3}$ gas. In this instance, for the higher transition data, an unresolved line detection from APEX was used, while the $N=3-2$ emission was resolved by the SMA with a resulting line ratio estimation of 0.73 (plus $10 \%$ errors). Our higher resolution data finds a much greater line flux in the $N=4-3$ transition by a factor of 3 (see disk integrated flux in Table 1), which is different from that estimated by Kastner et al. (2015), presumably due to beam dilution.

Thus the flux density ratio is increased, with a value of ${ }^{7}$ :

$$
\frac{N=4-3, J=\frac{9}{2}-\frac{7}{2}, F=4-3}{N=3-2, J=\frac{9}{2}-\frac{7}{2}, F=4-3}=2.16 \pm 0.45 .
$$

We have therefore re-analyzed the excitation characteristics of this emission using RADEX (van der Tak et al. 2007) and the state to state collision rates by Spielfiedel et al. (2012). This analysis is shown in Figure 12. With this revision, it seems clear that the emission arises from gas with at least moderate density $>10^{6} \mathrm{~cm}^{-3}$. If applicable to $\mathrm{C}_{2} \mathrm{H}$, this is consistent with fairly high densities. At face value, the observed ratio is also better fit by emission within warmer gas ( 40-50 K).

We have also explored solutions including a dust rich background source, as the $\mathrm{C}_{2} \mathrm{H}$ emission lies in front of the central dust rich midplane emitting at a temperature below $20 \mathrm{~K}$ at these radii (Andrews et al. 2016). This compresses the dependences at low densities (for all temperatures) and low temperatures (for all densities) but does not change the conclusion: $\mathrm{C}_{2} \mathrm{H}$ appears to be emitting from gas with densities greater than $10^{6} \mathrm{~cm}^{-3}$ consistent with our predictions. From Figure 11 we would predict that the outer $\mathrm{C}_{2} \mathrm{H}$ ring in DM Tau is emitting from gas that has lower density $\left(3 \times 10^{4} \mathrm{~cm}^{-3}<n_{\mathrm{H}_{2}}<10^{6} \mathrm{~cm}^{-3}\right)$ and is colder $\left(T_{\mathrm{gas}} \sim 10-20 \mathrm{~K}\right)$.

\subsection{Photodissociation of Carbonaceous Grains as the Origin of Simple Hydrocarbons}

In this paper we have outlined a consistent picture of coupled dust + gas physical chemical evolution that leads to the creation of hydrocarbon rings. We have implicitly assumed that $\mathrm{C}_{2} \mathrm{H}$ is produced from carbon that was extracted from $\mathrm{CO}$, aided by the depletion of oxygen in water ice. On the other hand, as discussed by Kastner et al. (2015) the photo-ablation of aliphatic or aromatic hydrocarbon grains would be a ready source of carbon as carbon-rich grains contain nearly half of the cosmic abundance of carbon (Chiar et al. 2013; Jones et al. 2013). Moreover, if carbon grains are being destroyed in gas where water remains frozen, then the $\mathrm{C} / \mathrm{O}$ ratio can be $>1$.

To explore this issue we use a calculation of the equilibrium abundance of $\mathrm{C}_{2} \mathrm{H}$ if produced via photodissociation of hydrogenated amorphous carbon grains, one of the potential carriers of refractory carbon, and destroyed by atomic oxygen. There are no calculations of photodissociation channels of hydrogenated amorphous carbon grains $(\mathrm{a}-\mathrm{C}: \mathrm{H})$ that directly lead to $\mathrm{C}_{2} \mathrm{H}$. However, laboratory work and models of hydrogenated amorphous carbon photoablation and carbon chemistry by Alata et al. (2015) show that creation of other hydrocarbons $\left(\mathrm{CH}_{4}, \mathrm{C}_{2} \mathrm{H}_{2}\right.$ as examples) leads to an increase in the abundance of $\mathrm{C}_{2} \mathrm{H}$. For this purpose we adopt their total photodissociation rate regardless of the product. This is $k_{\mathrm{ph}}=2 \times 10^{-12} G_{0} \exp \left(-2.0 A_{V}\right) F_{i} \mathrm{~s}^{-1}$. For TW Hya, $G_{0}$

\footnotetext{
We have assumed $15 \%$ calibration uncertainty for both ALMA and SMA.
} 

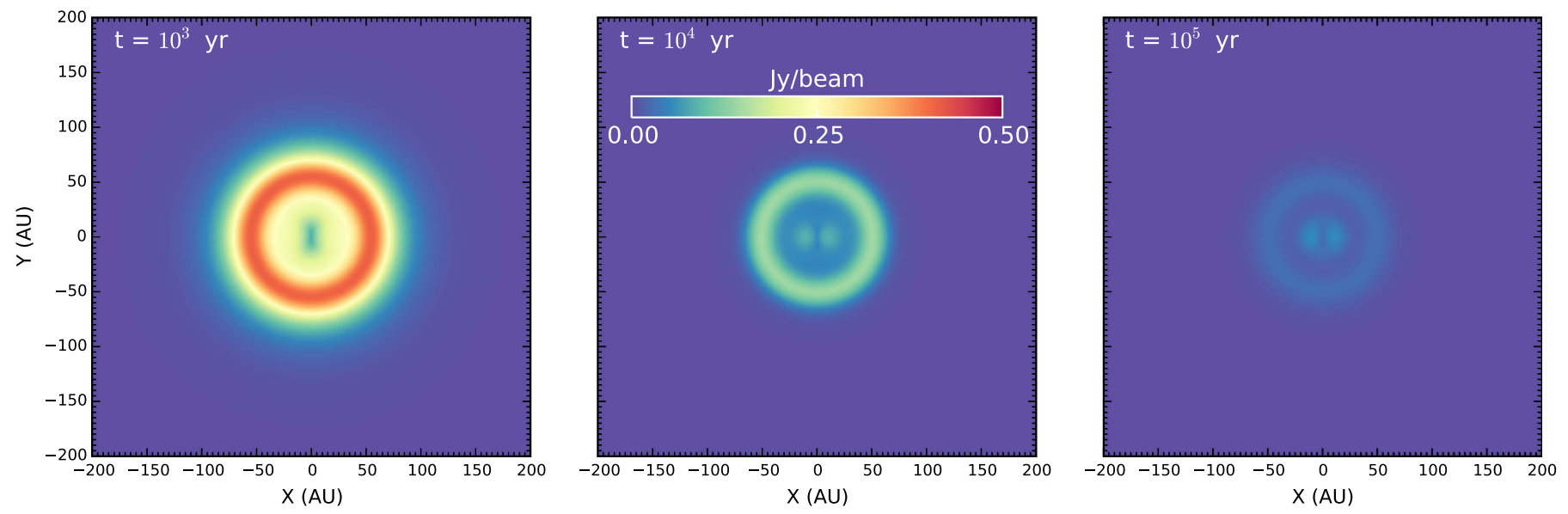

Figure 13. $\mathrm{C}_{2} \mathrm{H} J=7 / 2-5 / 2, F=4-3$ and $3-2$ emission at $10^{3}, 10^{4}$, and $10^{5}$ years, starting with a uniformly high $\mathrm{C}_{2} \mathrm{H}$ abundance of $10^{-5}$. The underlying disk structure is the one specified in Table 2 for TW Hya.

is $\sim 320$ at 100 au (Bergin et al. 2003), including the contribution from Ly $\alpha$ this would increase to 2000 (Herczeg et al. 2002; Bergin et al. 2004). $F_{i}$ is a factor that accounts for the fact that the parent a-C:H grain being ablated each time a photon is capable of breaking off a fragment. For simplicity we adopt $F_{i}=0.5$. The unshielded photorate is consistent with the calculation of polycyclic aromatic hydrocarbon (PAH) photodissociation producing $\mathrm{C}_{2}, \mathrm{C}_{2} \mathrm{H}$, or $\mathrm{C}_{2} \mathrm{H}_{2}$ fragments for a $\mathrm{PAH}$ consisting of $<20$ atoms (Visser et al. 2007). Thus, this calculation is roughly appropriate for PAHs, which are another potential solid state carbon carrier.

To obtain the maximum amount of $\mathrm{C}_{2} \mathrm{H}$ that might be created with this rate as the source term we further assume that $\mathrm{C}_{2} \mathrm{H}$ is the ultimate product of all dissociations. Under this case the equilibrium abundance will be as follows:

$$
\frac{d n_{\mathrm{C}_{2} \mathrm{H}}}{d t}=n_{\mathrm{a}-\mathrm{C}: \mathrm{H}} k_{\mathrm{ph}}-n_{\mathrm{C}_{2} \mathrm{H}} n_{\mathrm{O}} k_{\mathrm{O}, \mathrm{C}_{2} \mathrm{H}} \text {. }
$$

Here $k_{\mathrm{O}, \mathrm{C}_{2} \mathrm{H}}=10^{-10} \mathrm{~cm}^{3} \mathrm{~s}^{-1}$ is the destruction rate of $\mathrm{C}_{2} \mathrm{H}$ with oxygen atoms (assuming this is the main destruction channel). In steady state both right hand terms are equivalent and we can solve for the $\mathrm{C}_{2} \mathrm{H}$ abundance:

$$
x_{\mathrm{C}_{2} \mathrm{H}}=\frac{x_{\mathrm{a}-\mathrm{C}: \mathrm{H}} k_{\mathrm{ph}}}{x_{\mathrm{O}} n_{\mathrm{H}_{2}} k_{\mathrm{O}, \mathrm{C}_{2} \mathrm{H}}} .
$$

We assume that the abundance of carbon grains is $\sim 3 \times 10^{-7}$ relative to $\mathrm{H}$ and each grain carries $\sim 50$ carbon atoms as determined for PAHs (Tielens 2005). Strictly PAHs are not the same chemical form as a-C:H, but it provides a good baseline for the amount of carbon that might be present in the the solid state. We also assume that the distance is $60 \mathrm{au}, A_{V}=0.5^{\mathrm{m}}$ (or $\tau_{\mathrm{UV}}=1$ ), and a gas density of $10^{8} \mathrm{~cm}^{-3}$ which is the density and UV optical depth seen in the $\mathrm{C}_{2} \mathrm{H}$ layers in our models. Using these expressions if the abundance of oxygen atoms is near cosmic $\left(10^{-4}\right)$ then the equilibrium abundance of $\mathrm{C}_{2} \mathrm{H}$ would be $\sim 5 \times 10^{-10}$. Based on our simulations, this would be well below the value of $\sim 10^{-7}$ needed for detection. To be consistent with our results (with the $\mathrm{H}_{2}$ gas mass constrained by HD), the oxygen abundance would need to be reduced by 2-3 orders of magnitude. Furthermore, species such as PAHs are difficult to detect in $\mathrm{T}$ Tauri systems with the suggestion their abundances are reduced by 10-100 orders of magnitude (Geers et al. 2007; Akimkin et al. 2013); this would require even lower abundances of atomic oxygen. Thus, if carbonaceous grains are the source term for $\mathrm{C}_{2} \mathrm{H}$ emission, it also requires reduced abundances of oxygen in the disk atmosphere.

The timescale to reduce the grain by one small fragment is short and is only $\sim 100$ years. Depending on the size, small grains could be destroyed on timescales below $10^{5}$ yrs (Alata et al. 2015), but large grains or smaller grains in deeper layers would survive. However, the photorates of large ( $>25$ atoms) grains are significantly reduced by many orders of magnitude Visser et al. (2007) as there are more internal models to share the energy, as opposed to breaking a bond. Thus very large grains may not be the most viable source terms for $\mathrm{C}_{2} \mathrm{H}$ production.

If a-C:H or PAHs were the source term another question would be how long would $\mathrm{C}_{2} \mathrm{H}$ last in the gaseous state. Since our chemical network does not explicitly include PAH chemistry, we mimic the effect of PAH/a-C:H photodissociation by artificially setting the initial abundance of $\mathrm{C}_{2} \mathrm{H}$ to $10^{-5}$ (relative to hydrogen nuclei). This is consistent with the carbon grain abundance adopted above, assuming 50 carbon atoms which then uniformly produce $\mathrm{C}_{2} \mathrm{H}$. Otherwise this model assumes a normal $\mathrm{C} / \mathrm{O}$ ratio. We then evolve the system for $1 \mathrm{Myr}$ to see how the resulting emission in $\mathrm{C}_{2} \mathrm{H}$ changes with time.

Figure 13 shows the $\mathrm{C}_{2} \mathrm{H}$ emission map at $t=10^{3}, 10^{4}$, and $10^{5}$ years. Here the $\mathrm{C}_{2} \mathrm{H}$ emission decreases with time monotonically and only approaches observed values at early stages $\left(\sim 10^{3}\right.$ years). As in the discussion above longer timescales would be found for lower oxygen abundances. However, without a continuous supply of small PAH/a-C:H grains as a source of hydrocarbons and without depletion of oxygen, the carbon budget contained in the initial hydrocarbons (such as $\mathrm{C}_{2} \mathrm{H}$ ) will quickly be converted into other species (such as $\mathrm{CO}$ through oxygen production via $\mathrm{H}_{2} \mathrm{O}$ ice photodesorption). As noted in Kastner et al. (2015), the estimated PAH abundance is barely enough to account for the observed $\mathrm{C}_{2} \mathrm{H}$ emission, especially if we consider that the $\mathrm{PAH}$ abundance is usually argued to be already depleted in the disk environment (Akimkin et al. 2013). Thus, while destruction of carbon grains may be a source of carbon for $\mathrm{C}_{2} \mathrm{H}$, it is likely that the production of $\mathrm{C}_{2} \mathrm{H}$ is fueled by the extraction of carbon 
from $\mathrm{CO}$ and depletion of oxygen from $\mathrm{CO}$ and other major oxygen-bearing species (such as water and $\mathrm{CO}_{2}$ ). This is consistent with the measured depletion of CO in TW Hya at the very least (Favre et al. 2013; Nomura et al. 2016; Schwarz et al. 2016); we therefore predict that the outer disk of DM Tau should also exhibit a reduction in the abundance of volatile $\mathrm{CO}$, while the inner disk must have high carbon content to remain consistent with $\mathrm{C}^{18} \mathrm{O}$ measurements (Williams \& Best 2014).

\subsection{A Physical or a Chemical Effect?}

In our model we have associated dust evolution to two effects. One is physical, that is a redistribution of the UV opacity that increases the penetration of UV photons in the disk system. The other is the relative trapping of volatiles as icy mantles coating grains that settle, grow, and drift which induces an increase in the $\mathrm{C} / \mathrm{O}$ ratio. This brings up the question as to whether one can reproduce these observations with an increased C/O ratio alone. The work by Kama et al. (2016b) provides some insight to this issue as they explored the emission of several carbon-bearing species, including an unresolved observation of $\mathrm{C}_{2} \mathrm{H}$, in TW Hya. Similar to our result they find that reproducing the emission requires $\mathrm{C} / \mathrm{O}>1$. Their model does include a vertically stratified dust model that accounts for the settling of larger grains, but does not include the effects of grain drift. At face value Kama et al. (2016b) show that the total $\mathrm{C}_{2} \mathrm{H}$ flux can be reproduced by increasing the $\mathrm{C} / \mathrm{O}$ ratio.

In our models for TW Hya alone we require central carbon depletion to reproduce the emission distribution. Thus it would be hard to directly point out the effects of the UV field in this case. However, we have the two rings seen in DM Tau. The outer ring in particular requires both UV to power the chemistry and $\mathrm{C} / \mathrm{O}>1$. In addition, our models, for both sources, and Kama et al. (2016b) have a stronger UV field on the disk surface due to dust settling; in our case we find $G_{0} \geqslant 1$ in layers where $\mathrm{C}_{2} \mathrm{H}$ is forming. This, and the known association of $\mathrm{C}_{2} \mathrm{H}$ (and other hydrocarbons) with photodissociation regions (Pilleri et al. 2013; Nagy et al. 2015), argues that the UV field is also an important factor in hydrocarbon production.

\section{SUMMARY AND IMPLICATIONS}

We have presented high-resolution observations of $\mathrm{C}_{2} \mathrm{H}$ and $\mathrm{C}_{3} \mathrm{H}_{2}$ in the protoplanetary disks of TW Hya and DM Tau, two classical T Tauri stars. We summarize here key observational facts and results from a detailed analysis:

1. In TW Hya we resolve a single emission ring that peaks near 55 au in the submillimeter emission lines of both $\mathrm{C}_{2} \mathrm{H}$ and $c-\mathrm{C}_{3} \mathrm{H}_{2}$. For $\mathrm{C}_{2} \mathrm{H}$ the central emission hole is resolved with emission significantly weaker (by factor of 6) in the disk center than at ring peak. The peak emission in the hydrocarbon ring sits just outside of the resolved submillimeter dust disk.

2. The central hole of $\mathrm{C}_{2} \mathrm{H}$ in TW Hya requires depletion of carbon within $\sim 40 \mathrm{au}$, which is consistent with previous observations of $\mathrm{CO}$ isotopologues. We further rule out high dust optical depth as the cause of the ring structure, and require carbon depletion as the cause.

3. In DM Tau we detect two rings: an inner ring that is centered on a bright central peak in the submillimeter continuum emission (but not an edge) near $R \sim 50$ au and an outer ring at $350 \mathrm{au}$ near the edge of the detectable disk in submillimeter continuum emission.

4. We suggest that hydrocarbon rings are created via the coupling of the gas-phase chemistry to the spatial evolution of ice-coated dust grains. As ice-coated dust grains settle to the dust rich midplane they grow to larger sizes whereupon they drift toward smaller radii. This concentrates the dust mass in the midplane within the inner tens of au of the disk, with two attendant effects that are ingredients for the production of hydrocarbon rings. First, the redistribution of dust mass also redistributes the UV opacity, leaving the disk surface and outer disk essentially a UV-photon-dominated PDR. Second, it also concentrates the volatiles, with a greater effect for the more tightly bound $\mathrm{H}_{2} \mathrm{O}$ than the more volatile $\mathrm{CO}$, enhancing the gas-phase $\mathrm{C} / \mathrm{O}$ ratio to values $>1$ and increasing the hydrocarbon emission. This should readily deplete the outer disk of volatiles, which would therefore be the first "observable" effect. In the inner disk carbon could also be depleted due to formation of refractory grains or sequestration in larger bodies that are less susceptible to evaporation, which creates an inner hydrocarbon hole.

5. Using ancillary data, we perform an excitation analysis on the $\mathrm{C}_{2} \mathrm{H}$ emission showing that the emission must arise from gas with $n_{\mathrm{H}_{2}}>10^{6} \mathrm{~cm}^{-3}$. This is consistent with our model predictions.

6. We present parametric models that match the observed emission distribution in both systems to within a factor of 1.5. They illustrate the importance of chemical effects for hydrocarbon ring formation.

The presence of a hydrocarbon ring in two disk systems of disparate sizes and ages does not, by itself, provide evidence that hydrocarbon rings are a widespread feature of disk chemical evolution. Given the sensitivity of ALMA this question will be answered with a larger statistically significant sample. However, we suggest that these effects are likely prevalent. First, Kastner et al. (2014) performed an unbiased line survey between 275 and $357 \mathrm{GHz}$ of TW Hya and V4046 Sgr finding that $\mathrm{C}_{2} \mathrm{H}$ lines are among the strongest in the band (even brighter than ${ }^{13} \mathrm{CO}$ ). This hints at a significant $\mathrm{C}_{2} \mathrm{H}$ abundance enhancement. Second, given the coincidence of the $\mathrm{C}_{2} \mathrm{H}$ rings with the edge of the pebble disk, the production of hydrocarbon rings is most likely tied to dust settling, growth, and migration. These are common physical effects that have strong observational support in a wide range of systems (Guilloteau \& Dutrey 1998; Furlan et al. 2006; Isella et al. 2007; Piétu et al. 2007; Andrews et al. 2012). Thus we predict that hydrocarbon rings will be a common feature in disks, and the coupled gas-dust evolution is the single most important factor that influences the observable chemical signatures of gasrich disk systems.

This work was supported by funding from the National Science Foundation grant AST-1514670 and AST-1344133 (INSPIRE) along with NASA XRP grant NNX16AB48G. This paper makes use of the following ALMA data: ADS/JAO. ALMA\#2013.1.00198.S. ALMA is a partnership of ESO (representing its member states), NSF (USA) and NINS (Japan), together with NRC (Canada) and NSC and ASIAA (Taiwan) and KASI (Republic of Korea), in cooperation with the Republic of Chile. The Joint ALMA Observatory is 

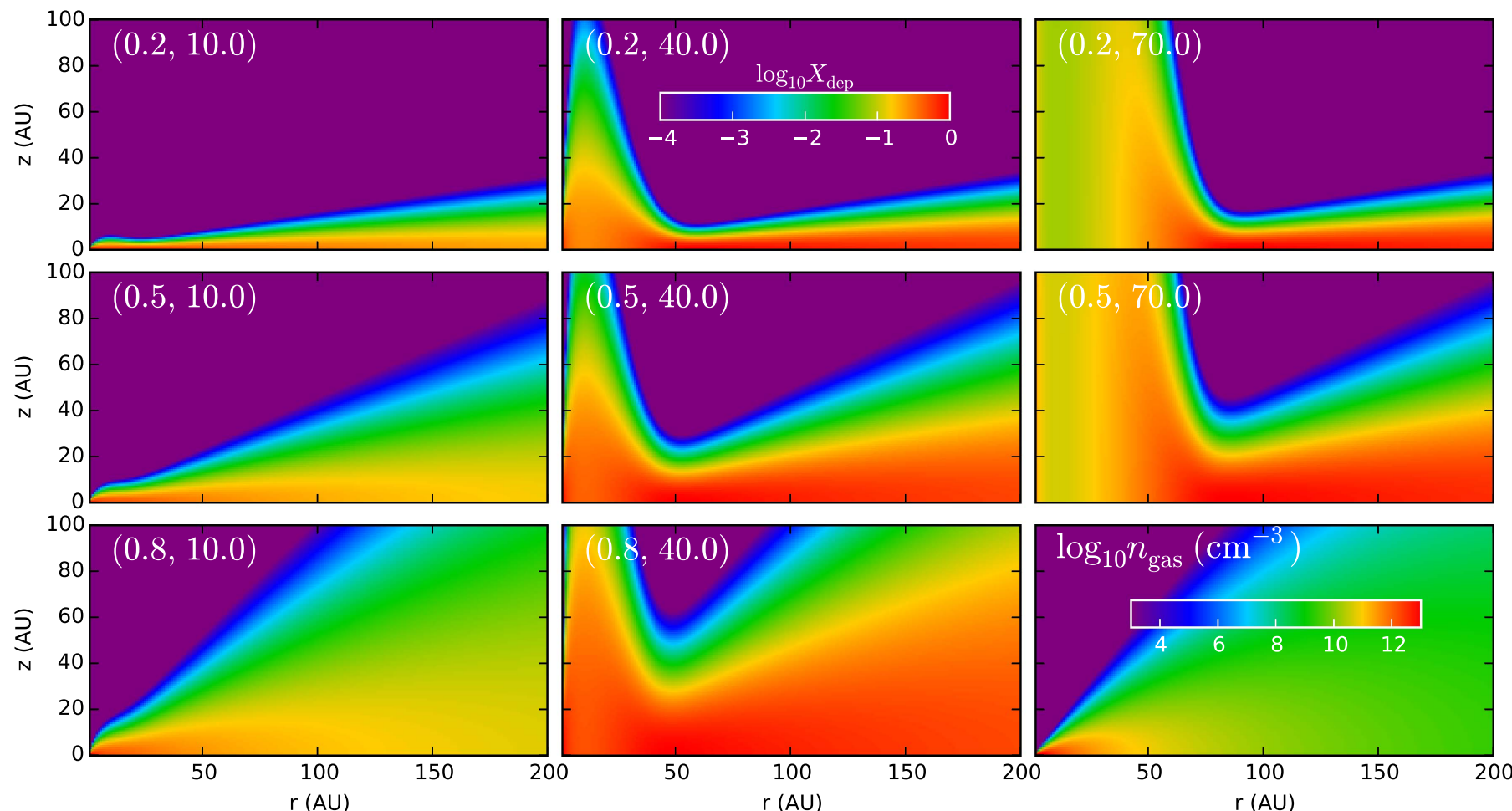

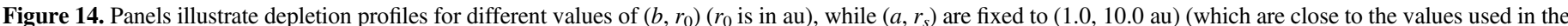

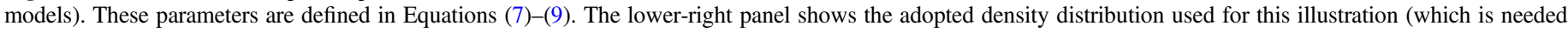

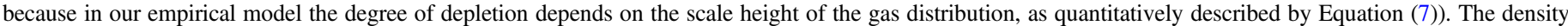

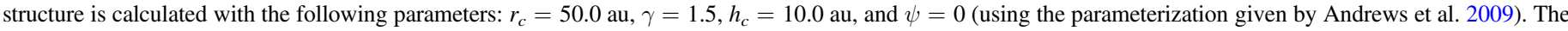
color scale of all panels are in log scale. For the elemental distribution, the highest value (in red) is to be interpreted as the non-depleted ISM abundance.

operated by ESO, AUI/NRAO and NAOJ. The National Radio Astronomy Observatory is a facility of the National Science Foundation operated under cooperative agreement by Associated Universities, Inc.

\section{APPENDIX A \\ EMPIRICAL APPROXIMATION FOR ELEMENTAL ABUNDANCE DISTRIBUTION}

To model the depletion of elements, we assume that the scale height for the abundance of the element under consideration is a fraction of the scale height of hydrogen at a given radius. Specifically, if the density of the hydrogen gas as a function of vertical height $z$ and radius $r$ is $n(z, r)$, then the abundance of the element to be depleted relative to its canonical value is taken to be

$$
X(z, r)=\left[\frac{n(z, r)}{n(z=0, r)}\right]^{f_{\mathrm{depl}}(r)} .
$$

For $f_{\text {depl }}$ we use the following form

$$
f_{\text {depl }}(r)=\frac{1}{y^{2}}-1,
$$

where

$$
y(r)=b+\frac{a-b}{2}\left[1-\tanh \left(\frac{r-r_{0}}{r_{\mathrm{s}}}\right)\right] .
$$

Here $a, b, r_{0}$, and $r_{\mathrm{s}}$ are parameters, with $a$ and $b$ being dimensionless. $a=y_{\max }=\left.y\right|_{r \rightarrow-\infty}$, and $b=y_{\min }=\left.y\right|_{r \rightarrow+\infty}$. $r_{0}$ marks the location outside of which the element is depleted, and within which may be enhanced, and $r_{\mathrm{s}}$ quantifies the width of the transition region. If $y>1$ (which may occur when $a>1), f_{\text {depl }}$ can take negative values, which means this parameterization allows the elemental distribution in the inner disk to increase with height above the disk. $f_{\text {depl }}=0$ means no depletion, and the larger $f_{\text {depl }}$, the more the element is depleted. To see the physical meaning of $f_{\text {depl }}$, assume $n(z, r) \propto \exp \left[-\frac{z^{2}}{2 h^{2}(r)}\right]$, where $h(r)$ is the scale height. Then we have

$$
X(z, r) \propto \exp \left\{-\frac{z^{2}}{2\left[h(r) / f_{\mathrm{depl}}^{1 / 2}(r)\right]^{2}}\right\} .
$$

So $f_{\text {depl }}^{1 / 2}$ is the ratio between the scale height of hydrogen gas and the scale heights of the abundances of oxygen or carbon. The calculated elemental abundances for carbon and oxygen are used as input to the thermo-chemical solver at each location of the disk. The parameters are chosen heuristically for each disk to investigate what $\mathrm{C}$ and $\mathrm{O}$ distribution best reproduce the observed $\mathrm{C}_{2} \mathrm{H}$ observations.

In Figure 14 we provide example distributions (in a relative sense) as to how this parameterization operates. Different panels correspond to different values of the $b$ and $r_{0}$ parameters, while $a$ is fixed to 1 and $r_{\mathrm{s}}$ fixed to $10 \mathrm{au}$. The lower-right panel is the gas density distribution used for the illustrations, which is needed per Equation (7). Basically, the smaller the $b$ parameter is, the more the element is vertically 

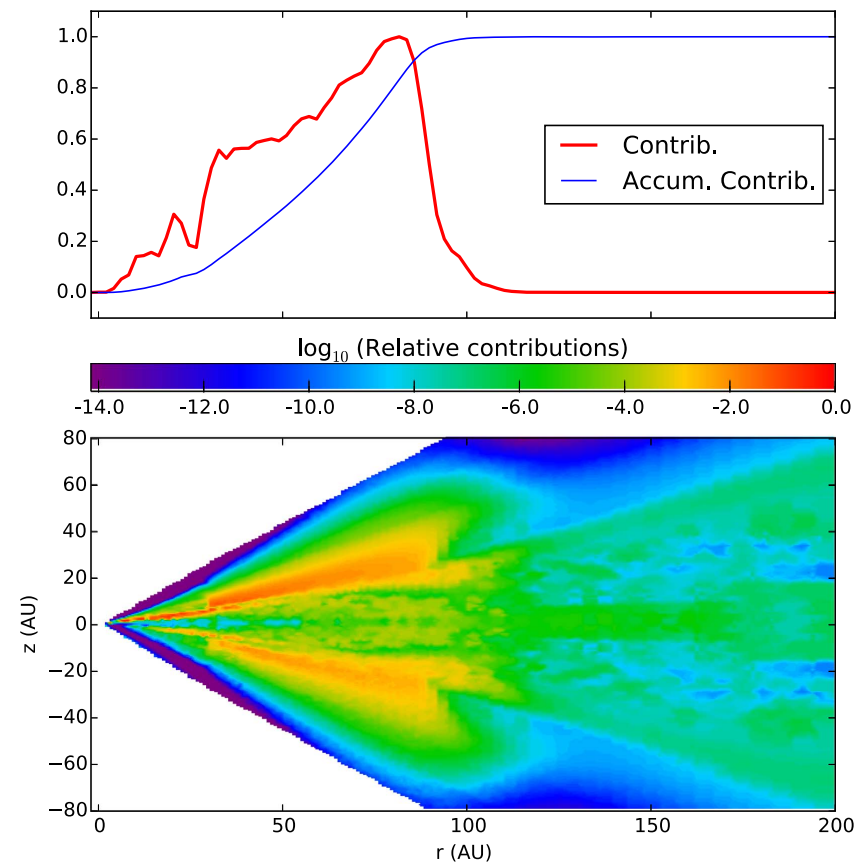

Figure 15. Top panel: contribution function at each radius (vertically integrated), and the radial accumulated contribution from the TW Hya model shown in Figures 9 and 10. Bottom panel: relative contribution to the $\mathrm{C}_{2} \mathrm{H}$ line emission as a function of position in the disk. Definitions described in Appendix.

depleted; and with a greater value for the $r_{0}$ parameter, the depletion will starts to occur at a greater distance, which means that $r_{0}$ is a kind of a "snow line" of the major carriers of the element under consideration.

\section{APPENDIX B \\ $\mathrm{C}_{2} \mathrm{H}$ CONTRIBUTION FUNCTION IN TW HYA}

We define the contribution of each location to the total emission to be the power emitted per unit length per unit area along the line of sight at that location that is actually received by a distant observer. From this intuitive definition, we see that the contribution function depends on the line of sight, and is a 3D function in general. Even if a location is very emissive, if it is behind high optical depth along the line of sight, its contribution will be low. Mathematically, the contribution function we use is

$$
\operatorname{contrib}(\boldsymbol{x})=j(\boldsymbol{x}) e^{-\tau_{\mathrm{los}}(\boldsymbol{x})},
$$

where $j(\boldsymbol{x})$ is the emission coefficient at $\boldsymbol{x}$, while $\tau_{\text {los }}(\boldsymbol{x})$ is the optical depth measured from $x$ to infinity along the line of sight.

For a disk with axisymmetry, the contribution function will not be axisymmetric unless the disk is viewed face on, in which case we can integrate it vertically (and times $r$ ) to get the radial contribution function, and then integrate radially to get the radial accumulative contribution.

Usually we only care about the relative importance of each location to the total observed emission, so we normalize the contribution function by dividing it with its maximum value.

\section{REFERENCES}

Aikawa, Y., \& Nomura, H. 2006, ApJ, 642, 1152

Aikawa, Y., Umebayashi, T., Nakano, T., \& Miyama, S. M. 1999, ApJ, 519,705

Aikawa, Y., van Zadelhoff, G. J., van Dishoeck, E. F., \& Herbst, E. 2002, A\&A, 386, 622

Akimkin, V., Zhukovska, S., Wiebe, D., et al. 2013, ApJ, 766, 8

Alata, I., Jallat, A., Gavilan, L., et al. 2015, A\&A, 584, A123

Andrews, S. M. 2015, PASP, 127, 961

Andrews, S. M., Wilner, D. J., Espaillat, C., et al. 2011, ApJ, 732, 42

Andrews, S. M., Wilner, D. J., Hughes, A. M., et al. 2012, ApJ, 744, 162

Andrews, S. M., Wilner, D. J., Hughes, A. M., Qi, C., \& Dullemond, C. P. 2009, ApJ, 700, 1502

Andrews, S. M., Wilner, D. J., Zhu, Z., et al. 2016, ApJ, 820, 40

Bergin, E., Calvet, N., D’Alessio, P., \& Herczeg, G. J. 2003, ApJL, 591, L159

Bergin, E., Calvet, N., Sitko, M. L., et al. 2004, ApJL, 614, L133

Bergin, E. A., Cleeves, L. I., Crockett, N., \& Blake, G. A. 2014, FaDi, 168, 61

Bergin, E. A., Cleeves, L. I., Gorti, U., et al. 2013, Natur, 493, 644

Bergin, E. A., Hogerheijde, M. R., Brinch, C., et al. 2010, A\&A, 521, L33

Bethell, T. J., \& Bergin, E. A. 2011, ApJ, 739, 78

Birnstiel, T., Klahr, H., \& Ercolano, B. 2012, A\&A, 539, A148

Blevins, S. M., Pontoppidan, K. M., Banzatti, A., et al. 2016, ApJ, 818, 22

Calvet, N., D’Alessio, P., Watson, D. M., et al. 2005, ApJL, 630, L185

Chapillon, E., Guilloteau, S., Dutrey, A., \& Piétu, V. 2008, A\&A, 488, 565

Chiang, E., \& Youdin, A. N. 2010, AREPS, 38, 493

Chiang, E. I., Joung, M. K., Creech-Eakman, M. J., et al. 2001, ApJ, 547, 1077

Chiar, J. E., Tielens, A. G. G. M., Adamson, A. J., \& Ricca, A. 2013, ApJ, 770,78

Cleeves, L. I., Adams, F. C., \& Bergin, E. A. 2013, ApJ, 772, 5

Cleeves, L. I., Bergin, E. A., Qi, C., Adams, F. C., \& Öberg, K. I. 2015, ApJ, 799, 204

D’Alessio, P., Calvet, N., Hartmann, L., Lizano, S., \& Cantó, J. 1999, ApJ, 527,893

Dartois, E., Dutrey, A., \& Guilloteau, S. 2003, A\&A, 399, 773

Debes, J. H., Jang-Condell, H., Weinberger, A. J., Roberge, A., \& Schneider, G. 2013, ApJ, 771, 45

Draine, B. T. 2006, ApJ, 636, 1114

Draine, B. T., \& Lee, H. M. 1984, ApJ, 285, 89

Du, F., \& Bergin, E. A. 2014, ApJ, 792, 2

Du, F., Bergin, E. A., \& Hogerheijde, M. R. 2015, ApJL, 807, L32

Dullemond, C. P., \& Dominik, C. 2004, A\&A, 421, 1075

Dullemond, C. P., van Zadelhoff, G. J., \& Natta, A. 2002, A\&A, 389, 464

Dutrey, A., Guilloteau, S., \& Guelin, M. 1997, A\&A, 317, L55

Dutrey, A., Guilloteau, S., \& Simon, M. 2003, A\&A, 402, 1003

Espaillat, C., D’Alessio, P., Hernández, J., et al. 2010, ApJ, 717, 441

Favre, C., Cleeves, L. I., Bergin, E. A., Qi, C., \& Blake, G. A. 2013, ApJL, 776, L38

Fogel, J. K. J., Bethell, T. J., Bergin, E. A., Calvet, N., \& Semenov, D. 2011, ApJ, 726, 29

Furlan, E., Hartmann, L., Calvet, N., et al. 2006, ApJS, 165, 568

Furuya, K., \& Aikawa, Y. 2014, ApJ, 790, 97

Geers, V. C., van Dishoeck, E. F., Visser, R., et al. 2007, A\&A, 476, 279

Goldsmith, P. F., Heyer, M., Narayanan, G., et al. 2008, ApJ, 680, 428

Guilloteau, S., \& Dutrey, A. 1998, A\&A, 339, 467

Gundlach, B., \& Blum, J. 2015, ApJ, 798, 34

Habing, H. J. 1968, BAN, 19, 421

Hartmann, L., Ballesteros-Paredes, J., \& Bergin, E. A. 2001, ApJ, 562, 852

Hartmann, L., Calvet, N., Gullbring, E., \& D’Alessio, P. 1998, ApJ, 495, 385

Henning, T., Semenov, D., Guilloteau, S., et al. 2010, ApJ, 714, 1511

Herczeg, G. J., Linsky, J. L., Valenti, J. A., Johns-Krull, C. M., \& Wood, B. E. 2002, ApJ, 572, 310

Herczeg, G. J., Wood, B. E., Linsky, J. L., Valenti, J. A., \& Johns-Krull, C. M. 2004, ApJ, 607, 369

Hogerheijde, M. R., Bekkers, D., Pinilla, P., et al. 2016, A\&A, 586, A99

Hogerheijde, M. R., Bergin, E. A., Brinch, C., et al. 2011, Sci, 334, 338

Hollenbach, D., Kaufman, M. J., Bergin, E. A., \& Melnick, G. J. 2009, ApJ, 690, 1497

Hueso, R., \& Guillot, T. 2005, A\&A, 442, 703

Isella, A., Testi, L., Natta, A., et al. 2007, A\&A, 469, 213

Johansen, A., Oishi, J. S., Mac Low, M.-M., et al. 2007, Natur, 448, 1022

Jones, A. P., Fanciullo, L., Köhler, M., et al. 2013, A\&A, 558, A62

Kama, M., Bruderer, S., Carney, M., et al. 2016a, A\&A, 588, 108 
Kama, M., Bruderer, S., van Dishoeck, E. F., et al. 2016b, A\&A, 592, 83 Kastner, J. H., Hily-Blant, P., Rodriguez, D. R., Punzi, K., \& Forveille, T. 2014, ApJ, 793, 55

Kastner, J. H., Qi, C., Gorti, U., et al. 2015, ApJ, 806, 75

Kenyon, S. J., Dobrzycka, D., \& Hartmann, L. 1994, AJ, 108, 1872

Kitamura, Y., Momose, M., Yokogawa, S., et al. 2002, ApJ, 581, 357

Lynden-Bell, D., \& Pringle, J. E. 1974, MNRAS, 168, 603

Martín-Doménech, R., Muñoz Caro, G. M., Bueno, J., \& Goesmann, F. 2014, A\&A, 564, A8

Mathis, J. S., Rumpl, W., \& Nordsieck, K. H. 1977, ApJ, 217, 425

McClure, M. K., Bergin, E. A., Cleeves, L. I., et al. 2016, ApJ, in press

McClure, M. K., Furlan, E., Manoj, P., et al. 2010, ApJS, 188, 75

Menu, J., van Boekel, R., Henning, T., et al. 2014, A\&A, 564, A93

Mumma, M. J., \& Charnley, S. B. 2011, ARA\&A, 49, 471

Nagy, Z., Ossenkopf, V., Van der Tak, F. F. S., et al. 2015, A\&A, 578, A 124

Nomura, H., Tsukagoshi, T., Kawabe, R., et al. 2016, ApJL, 819, L7

Öberg, K. I., Boogert, A. C. A., Pontoppidan, K. M., et al. 2011a, ApJ, 740, 109

Öberg, K. I., Murray-Clay, R., \& Bergin, E. A. 2011b, ApJL, 743, L16

Panić, O., Hogerheijde, M. R., Wilner, D., \& Qi, C. 2009, A\&A, 501, 269

Pérez, L. M., Chandler, C. J., Isella, A., et al. 2015, ApJ, 813, 41

Piétu, V., Dutrey, A., \& Guilloteau, S. 2007, A\&A, 467, 163

Pilleri, P., Treviño-Morales, S., Fuente, A., et al. 2013, A\&A, 554, A87

Piso, A.-M. A., Öberg, K. I., Birnstiel, T., \& Murray-Clay, R. A. 2015, ApJ, 815,109

Qi, C., Öberg, K. I., Wilner, D. J., et al. 2013, Sci, 341, 630

Raassen, A. J. J. 2009, A\&A, 505, 755

Reboussin, L., Wakelam, V., Guilloteau, S., Hersant, F., \& Dutrey, A. 2015, A\&A, 579, A82

Schindhelm, E., France, K., Herczeg, G. J., et al. 2012, ApJL, 756, L23

Schlafly, E. F., Green, G., Finkbeiner, D. P., et al. 2014, ApJ, 786, 29
Schneider, G., Silverstone, M. D., Hines, M. D., et al. 2005, in Protostars and Planets V, ed. B. Reipurth, D. Jewitt, \& K. Keil (Tuscon, AZ: Univ. Arizona Press) poster 8540

Schwarz, K. R., Bergin, E. A., Cleeves, L. I., et al. 2016, ApJ, 823, 91

Semenov, D., \& Wiebe, D. 2011, ApJS, 196, 25

Simon, M., Dutrey, A., \& Guilloteau, S. 2000, ApJ, 545, 1034

Spielfiedel, A., Feautrier, N., Najar, F., et al. 2012, MNRAS, 421, 1891

Tachihara, K., Neuhäuser, R., \& Fukui, Y. 2009, PASJ, 61, 585

Thi, W.-F., Mathews, G., Ménard, F., et al. 2010, A\&A, 518, L125

Tielens, A. G. G. M. 2005, The Physics and Chemistry of the Interstellar Medium (Cambridge: Cambridge Univ. Press)

Tielens, A. G. G. M., \& Hollenbach, D. 1985, ApJ, 291, 722

Tsukagoshi, T., Momose, M., Saito, M., et al. 2015, ApJL, 802, L7

Tsukagoshi, T., Nomura, H., Muto, T., et al. 2016, ApJL, 829, L35

Vacca, W. D., \& Sandell, G. 2011, ApJ, 732, 8

van der Tak, F. F. S., Black, J. H., Schöier, F. L., Jansen, D. J., \& van Dishoeck, E. F. 2007, A\&A, 468, 627

van Leeuwen, F. 2007, A\&A, 474, 653

Visser, R., Geers, V. C., Dullemond, C. P., et al. 2007, A\&A, 466, 229

Weidenschilling, S. J., \& Cuzzi, J. N. 1993, in Protostars and Planets III, ed. E. H. Levy \& J. I. Lunine (Tuscon, AZ: Univ. Arizona Press), 1031

Weinberger, A. J., Becklin, E. E., Schneider, G., et al. 2002, ApJ, 566, 409

Whipple, F. L. 1972, in 21st Nobel Symp. Proc., From Plasma to Planet, ed. A. Elvius (New York: Wiley), 211

Whittet, D. C. B. 2010, ApJ, 710, 1009

Williams, J. P., \& Best, W. M. J. 2014, ApJ, 788, 59

Yang, H., Herczeg, G. J., Linsky, J. L., et al. 2012, ApJ, 744, 121

Youdin, A. N., \& Kenyon, S. J. 2013, in From Disks to Planets, ed. T. D. Oswalt, L. M. French, \& P. Kalas (Dordrecht: Springer)

Zhang, K., Bergin, E. A., Blake, G. A., et al. 2016, ApJL, 818, L16

Zhang, K., Pontoppidan, K. M., Salyk, C., \& Blake, G. A. 2013, ApJ, 766,82 